\title{
Adhesion: role of bulk viscoelasticity and surface roughness
}

\author{
B Lorenz ${ }^{1,4}$, B A Krick ${ }^{2}$, N Mulakaluri ${ }^{1}$, M Smolyakova ${ }^{1}$, S Dieluweit ${ }^{3}$, \\ W G Sawyer ${ }^{2}$ and B N J Persson ${ }^{1,4}$ \\ ${ }^{1}$ PGI, FZ-Jülich, D-52425 Jülich, Germany \\ 2 Department of Mechanical and Aerospace Engineering, University of Florida, Gainesville, FL 32611, \\ USA \\ ${ }^{3}$ ICS-7, FZ-Jülich, D-52425 Jülich, Germany \\ E-mail: b.persson@fz-juelich.de
}

Received 4 January 2013, in final form 5 April 2013

Published 7 May 2013

Online at stacks.iop.org/JPhysCM/25/225004

\begin{abstract}
We study the adhesion between smooth polydimethylsiloxane (PDMS) rubber balls and smooth and rough poly(methyl methacrylate) (PMMA) surfaces, and between smooth silicon nitride balls and smooth PDMS surfaces. From the measured viscoelastic modulus of the PDMS rubber we calculate the viscoelastic contribution to the crack-opening propagation energy $\gamma_{\text {eff }}(v, T)$ for a wide range of crack tip velocities $v$ and for several temperatures $T$. The Johnson-Kendall-Roberts (JKR) contact mechanics theory is used to analyze the ball pull-off force data, and $\gamma_{\operatorname{eff}}(v, T)$ is obtained for smooth and rough surfaces. We conclude that $\gamma_{\mathrm{eff}}(v, T)$ has contributions of similar magnitude from both the bulk viscoelastic energy dissipation close to the crack tip, and from the bond-breaking process at the crack tip. The pull-off force on the rough surfaces is strongly reduced compared to that of the flat surface, which we attribute mainly to the decrease in the area of contact on the rough surfaces.
\end{abstract}

(Some figures may appear in colour only in the online journal)

\section{Introduction}

The origin of adhesion between macroscopic bodies is a very important but complex topic [1-5]. Even the weakest force of interest in condensed matter physics, namely the van der Waals interaction, is very strong on a macroscopic scale. Thus, the force necessary to separate two flat surfaces with $\sim 1 \mathrm{~cm}^{2}$ cross section, bound together with the van der Waals interaction, corresponds to the weight of a car [6]. However, such strong adhesive forces are almost never observed in real-life situations, as is vividly evident, e.g., by the fact that we are able to walk or drive a car on a road. The reason that adhesion is usually not observed between macroscopic solid objects, a phenomenon often referred to as the adhesion paradox [1], is the ever present surface roughness (and contamination films) on solid bodies. Thus, because of surface roughness, solid objects will in general only make contact at a small fraction of the nominal or apparent contact

4 www.MultiscaleConsulting.com. area. The small area of real contact, and the elastic energy stored in the vicinity of the asperity contact regions (due to the elastic deformations of the solids), will reduce the pull-off force to almost zero in most practical cases. For elastic solids the stored interfacial elastic energy is, at least in part, 'given back' during pull-off and helps to break the interfacial bonds between the solids. In addition, during pull-off, a stress concentration will occur at the edges of the contact regions so that the interfacial bonds will not break uniformly, but rather via interfacial crack propagation, which will occur at a much smaller pull-off force than would be observed if all the interfacial bonds were to break simultaneously.

Strong adhesion requires very smooth surfaces and/or that at least one of the solids is elastically very soft, e.g., rubber. In this case the area of real contact may be similar to the nominal contact area, and the elastic deformation energy stored up at the interface is small enough not to severely reduce the adhesion. In a classical study Fuller and Tabor [7] studied the adhesion between rubber balls and hard rough substrate surfaces with different root-mean-square 

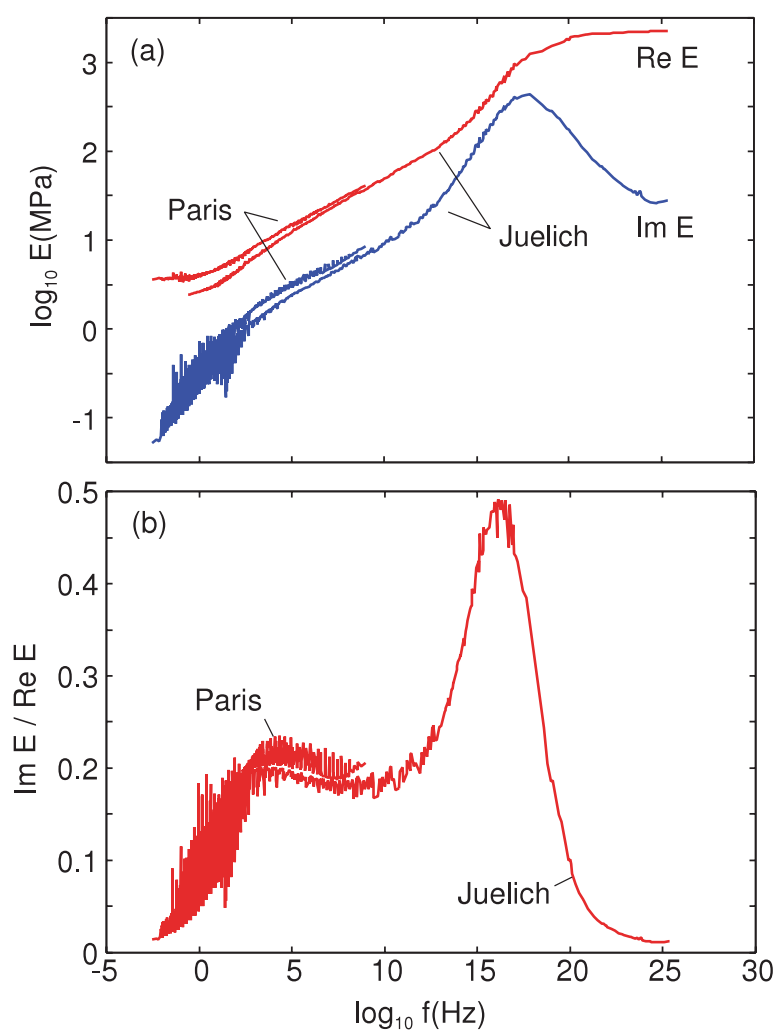

Figure 1. (a) The real and imaginary part of the viscoelastic modulus, and (b) $\tan \delta$ for PDMS as a function of frequency at room temperature $T=20^{\circ} \mathrm{C}$. The curves denoted Jülich and Paris were obtained by us and by the authors of [17], respectively.

(rms) roughness amplitude $h_{\text {rms }}$. For 'normal' rubber, with a low-frequency Young's elastic modulus of the order of a few $\mathrm{MPa}$, they found that even an rms roughness $h_{\mathrm{rms}} \approx 1 \mu \mathrm{m}$ results in a vanishing pull-off force. The authors did not present any detailed quantitative analysis of the experimental data, which would require knowledge of the substrate surface roughness power spectrum $C(q)$, which they did not measure. In addition, even silicon rubber, as used in their study, is not a purely elastic material but exhibits some viscoelasticity which will result in a pull-off force which depends on the pull-off velocity. Furthermore, the bond-breaking process at the interface will also depend on the separation velocity (and the temperature) as it involves stress-aided thermally activated processes [8-15].

In this paper we present a detailed study of the adhesion of PDMS rubber to smooth and rough surfaces. We have fully characterized the rubber (viscoelastic modulus $E(\omega)$ ) and the surface topography (surface roughness power spectrum $C(q)$ ) and present a quantitative comparison between theory and experiments. We find that the effective interfacial crack propagation energy, $\gamma_{\mathrm{eff}}(v, T)=\gamma_{0}(v, T)[1+f(v, T)]$, depends on the velocity due to both viscoelastic bulk energy dissipation close to the crack tip (factor $[1+f(v, T)]$ ), and due to the velocity dependence of the bond-breaking processes at the crack tip (factor $\gamma_{0}(v, T)$ ). We find, in agreement with Fuller and Tabor [7] and others [16], that even a slight surface roughness can result in a nearly vanishing pull-off force. We analyzed the pull-off data from experiments on the rough surfaces using the contact mechanics theory of Persson.

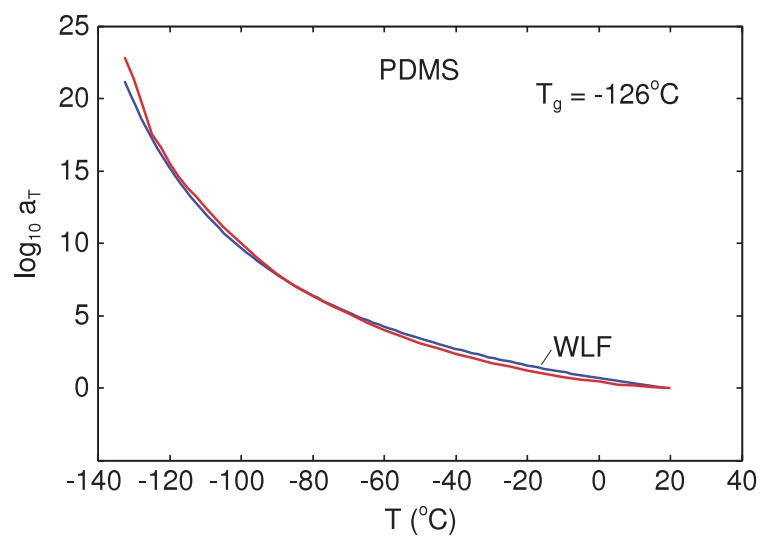

Figure 2. Red curve: the shift factor obtained by shifting the frequency segments to obtain the master curve shown in figure 1 . Blue curve: the WLF result $a_{T}=-A\left[\left(T-T_{\mathrm{g}}\right) /\left(B+T-T_{\mathrm{g}}\right)-\right.$ $\left.\left(T_{0}-T_{\mathrm{g}}\right) /\left(B+T_{0}-T_{\mathrm{g}}\right)\right]$, where $T_{\mathrm{g}}=-126^{\circ} \mathrm{C}$ is the glass transition temperature, $T_{0}=20^{\circ} \mathrm{C}$ the reference temperature, $A=24.0$ and $B=55.6^{\circ} \mathrm{C}$.

\section{Experimental details}

In section 2.1 we describe how we obtained the viscoelastic modulus $E(\omega)$ for the rubber used in our adhesion experiments. Section 2.2 describes 1D-stylus topography measurements and the surface roughness power spectra $C(q)$ of the surfaces used in some of the adhesion experiments. Finally in section 2.3 we describe the two sets of adhesion experiments we have performed.

\subsection{Viscoelastic modulus}

To calculate the viscoelastic factor $[1+f(v, T)]$ in the expression for the energy per unit area for the (opening) crack propagation, we need the rubber bulk viscoelastic modulus $E(\omega)$. From the viscoelastic modulus measured at small strain $(0.2 \%)$ for 12 frequency points between $f=0.25$ and $28.0 \mathrm{~Hz}$, and for many temperatures between $T=-130$ and $20^{\circ} \mathrm{C}$, we have obtained a smooth master curve by shifting the frequency segments of the real part of $E(\omega)$. The results for Re $E(\omega)$ and $\operatorname{Im} E(\omega)$ are shown (without smoothing) in figure 1. In the same figure we show the results obtained by Nguyen et al in a narrower frequency range [17]. The corresponding shift function $a_{T}$ is shown in figure 2 (red line). The blue line in the same figure is the Williams-Landel-Ferry (WLF) [18] result $a_{T}=-A\left[\left(T-T_{\mathrm{g}}\right) /\left(B+T-T_{\mathrm{g}}\right)-\left(T_{0}-T_{\mathrm{g}}\right) /\left(B+T_{0}-T_{\mathrm{g}}\right)\right]$, where $T_{\mathrm{g}}=-126^{\circ} \mathrm{C}$ is the glass transition temperature, $T_{0}=$ $20^{\circ} \mathrm{C}$ the reference temperature, $A=24.0$ and $B=55.6^{\circ} \mathrm{C}$.

\subsection{Surface roughness power spectrum}

In section 4.5 we study the adhesion between silicon rubber balls and smooth (flat) and sandblasted PMMA substrate surfaces. We have measured the surface topography of the PDMS surfaces using stylus (Talysurf 120 from Taylor-Hobson) 1D-line scans. Figure 3 shows the power spectra $[6,19]$,

$$
C(q)=\frac{1}{(2 \pi)^{2}} \int \mathrm{d}^{2} x\langle h(\mathbf{x}) h(\mathbf{0})\rangle \mathrm{e}^{\mathrm{i} \mathbf{q} \cdot \mathbf{x}},
$$




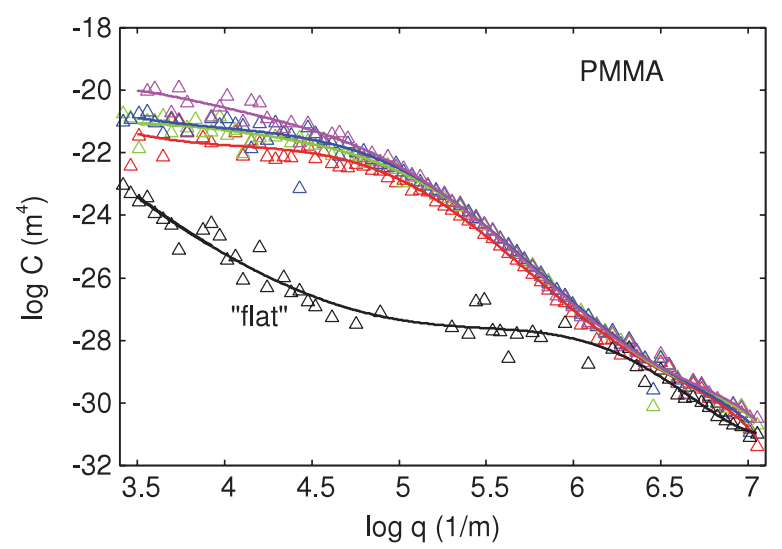

Figure 3. The surface roughness power spectrum of four sandblasted PMMA surfaces and one smooth (not sandblasted) PMMA surface. The substrates were sandblasted (from bottom to top): 0, 2, 6, 9 and $13 \mathrm{~min}$. The solid lines are a seventh order polynomial fit to the experimental data.

of the PMMA substrates used in the adhesion tests. The solid lines are seventh order polynomial fits to the experimental data. The 'smooth' surface and the four rough surfaces 1 , 2,3 and 4 have rms roughness values $0.379 \mu \mathrm{m}$ (smooth), $1.49,2.09,2.58$ and $3.18 \mu \mathrm{m}$, and rms-slopes $0.1,0.22,0.30$, 0.30 and 0.31 , respectively. The rough surfaces were prepared by sandblasting the PMMA substrate for different amounts of time: $2,6,9$ and $13 \mathrm{~min}$, resulting in the four surfaces $1,2,3$ and 4 , respectively. Note that the short wavelength (large wavevector $q$ ) roughnesses on all the rough surfaces are nearly the same, but the roll-off wavevector decreases as the sandblasting time increases, i.e., the roughness extends to longer and longer wavelengths as the sand blasting time increases. This is indeed the expected behavior, and simple theories [20] suggest that the roll-off wavevector scales as $t^{-1 / z}$ with the sandblasting time $t$, where $z$ is the so-called dynamical exponent. This result is consistent with the experimental observations. Since the rms-slope is determined mainly by the large wavevector surface roughness components, and since that region in the surface roughness power spectrum is very similar for the three most rough surfaces, it explains why the rms-slope values are so similar for these surfaces.

\subsection{Adhesion experiments}

We have studied the adhesional interaction between PDMS balls and PMMA, and between silicon nitride balls and PDMS, using two different types of adhesion experiments involving adhesion at different length scales.

In the first experiment (performed in Jülich) we bring a PDMS half sphere with radius $R=46.5 \mathrm{~mm}$ into contact with a PMMA substrate, as shown in figure 4. The PMMA substrate is either smooth or has different amounts of surface roughness. It is positioned on a very accurate balance (Excellence XA205DU Analytical Balance produced by Mettler Toledo), which has a reproducibility of $0.01 \mathrm{mg}$ (or $\approx 0.1 \mu \mathrm{N}$ ) at low loads. After zeroing the scale of the

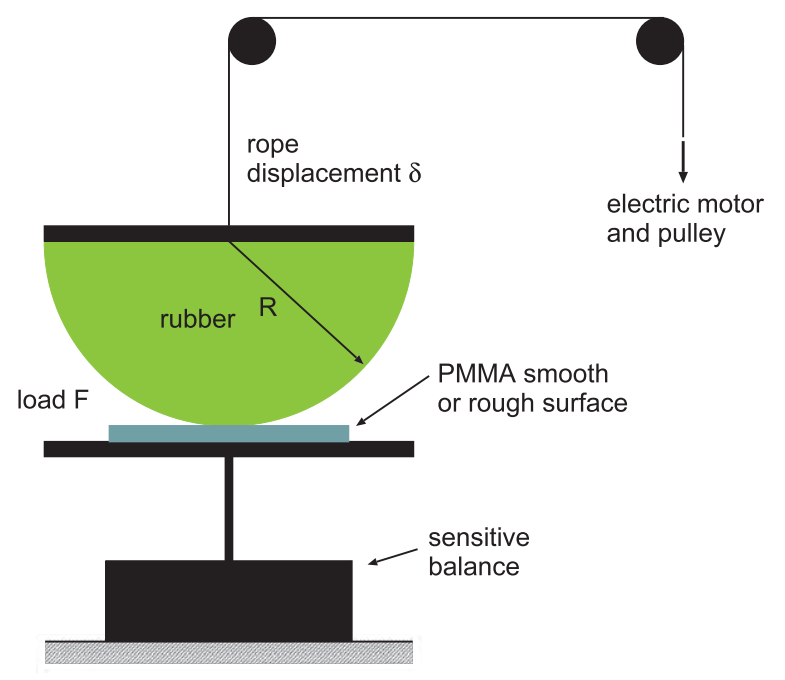

Figure 4. The Jülich experimental setup for measuring adhesion.

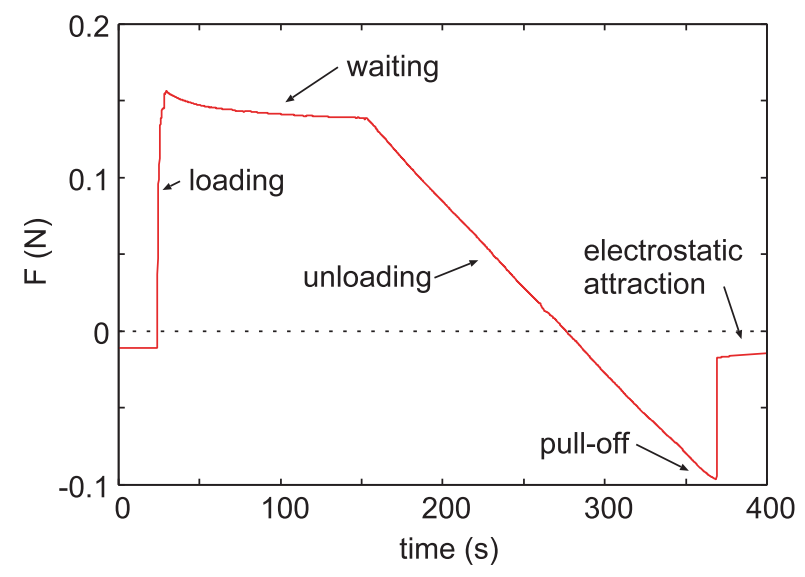

Figure 5. A loading-unloading cycle of a PDMS sphere (radius $R=46.5 \mathrm{~mm}$ ) against a smooth PMMA surface. The pull-off velocity $v_{z} \approx 1 \mathrm{~mm} \mathrm{~s}^{-1}$. The pull-off force $\approx 0.1 \mathrm{~N}$ corresponds to the effective (JKR) interfacial energy $\gamma_{\mathrm{eff}}=0.45 \mathrm{~J} \mathrm{~m}^{-2}$, which is $\sim 9$ times higher than expected for infinitesimally slow pull-off assuming van der Waals bonding between the rubber and the PMMA substrate.

instrument we can measure the force on the substrate as a function of time. The PDMS half sphere is brought into contact with the substrate and loaded with different preloads for a certain time period. After this waiting period the ball is slowly moved upwards with a constant velocity, resulting in an unloading of the substrate, as can be seen in figure 5, which shows a typical force-time curve as a result of the procedure described above. Because of adhesion between the half sphere and the substrate the measured force becomes negative in the unloading cycle until the contact is broken and the PDMS sphere pulled off from the substrate. To move the PDMS half sphere upwards we have used an electric motor coiling up a nylon cord, which is glued to the glass plate to which the upper flat surface of the PDMS probe was attached. The pulling velocity can be changed directly by the motor or by attaching the cord to different gear wheels in a transmission. 
The lowest velocity reported on below was realized by adding a soft rubber band to the nylon cord.

Also shown in figure 5 is the electrostatic interaction between the two solids before and after contact. This is why the force signal does not continue to zero when the sample is pulled off or before it is brought into contact. Note that this is an exaggerated example, and in the experiment this effect was negligible after cleaning the samples (see also appendix A). Nevertheless, the contribution to adhesion from charging is an interesting but not well understood problem which deserves more attention [21, 22].

The PDMS half sphere was produced by molding Sylgard 184 against a smooth glass sphere. This is a two-component kit purchased from Dow Corning (Midland, MI), consisting of a base (vinyl-terminated polydimethylsiloxane) and a curing agent (methylhydrosiloxane-dimethylsiloxane copolymer) with a suitable catalyst. From these two components we prepared a mixture of 10:1 (base/cross-linker) in weight. The mixture was degassed to remove the trapped air induced by stirring from the mixing process and then poured into the cast. The samples were cured in an oven at $80^{\circ} \mathrm{C}$ for $14 \mathrm{~h}$.

However, even after curing, the samples still have free polymer chains in the bulk that can move (diffuse) to the surface of the PDMS sphere and hence influence the adhesional interaction. To remove a large fraction of these free chains the samples were swollen in hexane for $24 \mathrm{~h}$ at room temperature $\left(\approx 20^{\circ} \mathrm{C}\right)$. The solvent was then replaced and the procedure repeated twice. To document the influence on the PDMS we have measured the weight before and after this procedure and found a mass reduction of $\approx 3.6 \%$ for all samples. The (low-frequency) Young's Modulus can be deduced if one measures the dimensions of the sample in the swollen state and after shrinkage. From this we estimate the elastic modulus $E \approx 2.2 \mathrm{MPa}$, while in a separate compression experiment we measured $2.3 \mathrm{MPa}$.

The second experiment (at the University of Florida) involves microadhesion experiments with in situ contact observation. An optical in situ microtribometer [23, 24] was used for micro-scale adhesion experiments. The tribometer (see figure 6(a)) was used to perform load-unload experiments between polished silicon nitride spheres (with radii $R \approx$ 3.2, 2.4 and $1.6 \mathrm{~mm}$ ) and flat, transparent Sylgard 10:1 PDMS elastomer coated on glass optical windows 5 . In this experiment we did not remove the free PDMS chains. The sphere is fixed to the end of a cantilever force transducer, whose deflection is monitored by capacitance probes. The probe is lowered in to and raised out of contact using a piezoelectric stage with $0.4 \mathrm{~nm}$ resolution and $1 \mathrm{~nm}$ repeatability. The spheres were loaded into the elastomer to approximately $1 \mathrm{mN}$, held for a relaxation time of $0.1,1,10$, or $100 \mathrm{~s}$, and then unloaded at prescribed piezoelectric stage rates of $0.1,1,10,100$ and $1000 \mu \mathrm{m} \mathrm{s}^{-1}$. The externally applied load is measured with a resolution of better than $1 \mu \mathrm{N}$, and is linearly proportional to the displacement of

5 The PDMS rubber film on the glass substrate in the UF experiment was $0.35 \mathrm{~mm}$ thick. As long as the film thickness is larger than the diameter of the contact area at pull-off (of the order of $0.1 \mathrm{~mm}$ ), it is effectively infinitely thick with respect to the contact mechanics involved, see [50].

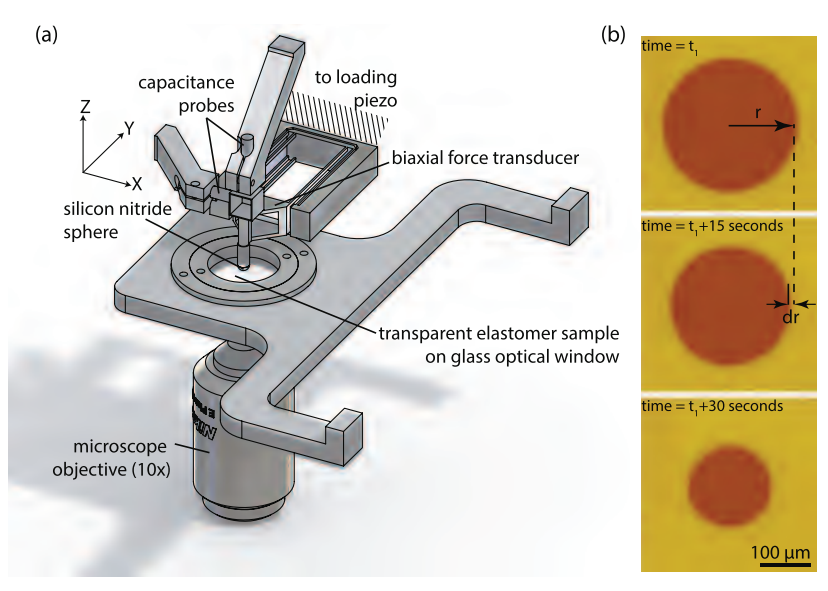

Figure 6. (a) The University of Florida experimental setup for measuring adhesion, and (b) optical images of the contact.

the capacitance probe; sphere penetration $\delta$ is monitored by subtracting the cantilever deflection from the piezoelectric stage position. Images of the contact between the silicon nitride spheres and the PDMS elastomer, see figure 6(b), are acquired at two images per second and synched with force and position data. The images are processed to calculate the actual contact area, contact half width or radius $r$ and the change in radius with respect to time $\mathrm{d} r / \mathrm{d} t$, see figure 7 for some examples. The maximum pull-off force and other adhesion parameters can be measured from the synched contact, force and displacement data.

\section{Energy dissipation at the opening crack}

The propagation of cracks in rubber is of fundamental importance for many applications, e.g., rubber wear [25], for pressure sensitive adhesives [26], and also for sliding or rolling friction [27]. The strength of adhesion and cohesion of elastomers can be characterized by the amount of energy $\gamma_{\text {eff }}$ required to advance the crack tip by one unit area. It has been shown experimentally that $\gamma_{\text {eff }}$ depends both on the crack tip velocity $v$ and the temperature $T$ and that [28-30]

$$
\gamma_{\mathrm{eff}}(v, T)=\gamma_{0}[1+f(v, T)] .
$$

Concerning interfacial (between the rubber and the substrate) crack propagation we consider the measured value of $\gamma_{\text {eff }}$ at extremely low crack velocities, which is of the order of $\approx 0.1 \mathrm{~J} \mathrm{~m}^{-2}$, while viscous effects in the rubber are negligible. This represents the energy needed to break the interfacial rubber-substrate bonds, which are usually of the van der Waals type, and is denoted as $\gamma_{0}$. For simple hydrocarbon elastomers, the effect of temperature can be completely accounted for by applying a simple multiplying factor, denoted by $a_{T}$, to the crack velocity $v$, i.e., $f(v, T)=$ $f\left(a_{T} v\right)$. Moreover, values of $a_{T}$ found experimentally are equal to the Williams-Landel-Ferry (WLF) [18] function determined from the temperature dependence of the bulk viscoelastic modulus, clearly proving that the large effects of crack velocity and temperature on crack propagation in rubber materials are due to viscoelastic processes in the bulk. 


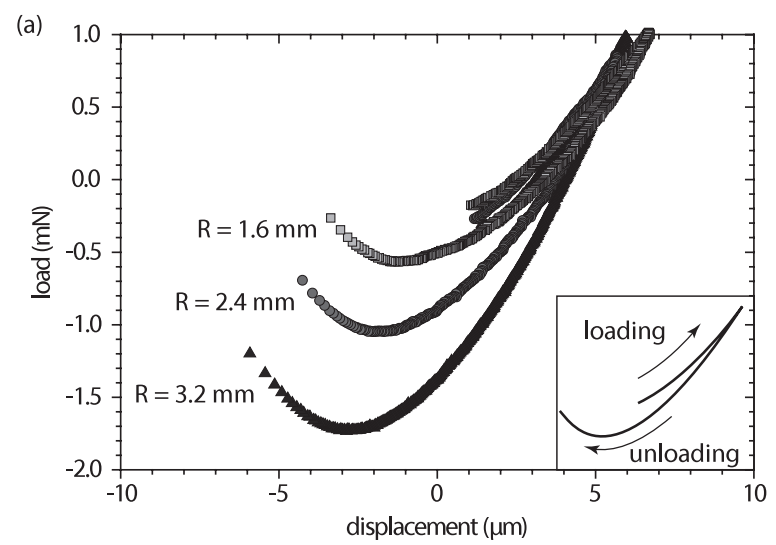

(b)

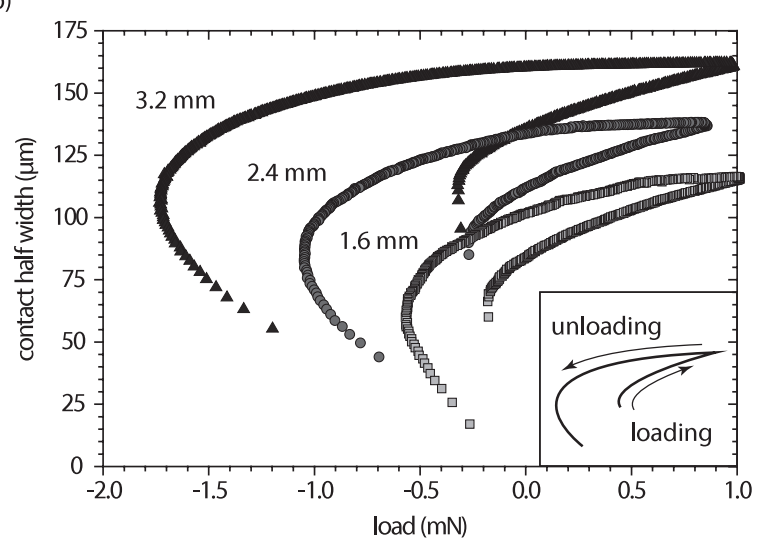

Figure 7. (a) The measured relation between the load and the vertical displacement $\delta$, and (b) between the contact radius $r$ and the load, for three different glass balls against PDMS elastomer.

In (1) the function $f(v, T)=f\left(a_{T} v\right)$ describes the bulk viscoelastic energy dissipation in front of the crack tip. This term is determined by the viscoelastic modulus $E(\omega)$ of the rubber, and can be calculated theoretically. The factor $\gamma_{0}$ is due to the bond breaking (in our applications between the rubber and the substrate) at the crack tip (in the so-called crack tip process zone), which may involve highly nonlinear processes. This term cannot be calculated theoretically, and must be deduced directly from experimental data. However, the strongest velocity dependence in (1) is in general derived from the factor $f(v, T)$, which may enhance $\gamma_{\text {eff }}$ by a factor $10^{3}$ or more at high crack tip velocities.

In $[31,32]$ we have shown that

$$
\gamma_{\mathrm{eff}}(v)=\gamma_{0}\left[1-\frac{2}{\pi} E_{0} \int_{0}^{2 \pi v / a} \mathrm{~d} \omega \frac{F(\omega)}{\omega} \operatorname{Im} \frac{1}{E(\omega)}\right]^{-1}
$$

where $E_{0}=E(0)$ and

$$
F(\omega)=\left[1-\left(\frac{\omega a}{2 \pi v}\right)^{2}\right]^{1 / 2}
$$

The crack tip radius $a=a(v)$ depends on the crack tip velocity $v$ (and temperature), and can be determined if one assumes that the stress at the crack tip takes some critical value $\sigma_{\mathrm{c}}$. This gives

$$
\frac{a}{a_{0}}=\frac{\gamma_{\text {eff }}}{\gamma_{0}}
$$

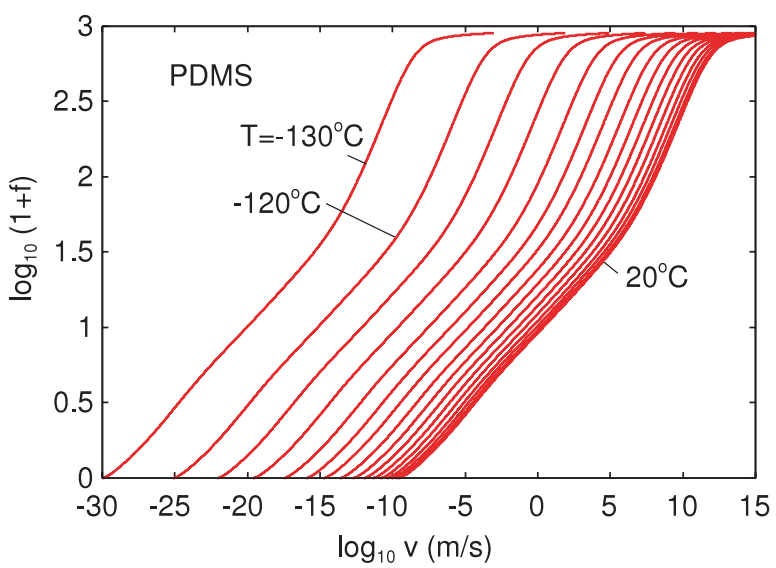

Figure 8. The logarithm (to base 10) of the crack propagation energy enhancement factor $\gamma_{\mathrm{eff}}(v) / \gamma_{0}=1+f$ as a function of the logarithm (to base 10) of the crack tip velocity for PDMS rubber without extracting free chains.

where $a_{0}$ is the crack tip radius for a very slowly moving crack. For high crack tip velocities $\gamma_{\text {eff }}(v) \approx \gamma_{0} E(\infty) / E(0) \gg$ $\gamma_{0}$. This is possible only if the denominator in (2) is close to zero for high crack tip velocities, which means that the term involving the integral must be close to unity. If (2) is used directly to calculate $\gamma_{\text {eff }}(v)$ numerically this requires that $E(\omega)$ is accurately known for all frequencies, which is usually not the case. However, it is possible to rewrite (2) in a form convenient for numerical calculations (see $[32,33]$ ).

In figure 8 we show the interfacial crack propagation viscoelastic energy enhancement factor $\gamma_{\mathrm{eff}}(v) / \gamma_{0}=[1+f]$ as a function of the logarithm of the crack tip velocity $v$ for PDMS rubber. This function $f(v, T)$ is calculated using (2)-(4) with $a_{0}=1 \mathrm{~nm}$, and using the viscoelastic modulus shown in figure 1.

For PDMS rubber, in a large frequency range, $\operatorname{Re} E(\omega) \sim \omega^{\beta}$ with $\beta \approx 0.12$. This is a much weaker frequency dependence than observed for most other rubbers, e.g., styrene-butadiene (SB) rubber, where $\beta \approx 0.4$. This has important implications for the bulk viscoelastic contribution $[1+f(v, T)]$ to the velocity dependence of the crack propagation energy $\gamma_{\text {eff }}$, which will scale as $[1+f(v, T)] \sim v^{\alpha}$ with $\alpha=\beta /(1+\beta)$. The velocity dependence of $\gamma_{\text {eff }}(v)$ has been measured by Gent for SB rubber [30]. He indeed found $\alpha \approx 0.27$, which is very close to the prediction $\alpha=\beta /(1+$ $\beta) \approx 0.28$ using $\beta \approx 0.4$. A good test of the crack propagation theory presented above is to find out experimentally if $\gamma_{\text {eff }}(v, T)$ indeed exhibits a weaker velocity dependence for PDMS than predicted for most other types of rubber. We will show in section 4.2 that for PDMS at small velocities $\gamma_{\text {eff }}(v, T) \sim v^{\alpha}$ with $\alpha \approx 0.12$, in good agreement with the theory prediction $\alpha=\beta /(1+\beta) \approx 0.11$ using $\beta \approx 0.12$.

\section{Adhesion}

\subsection{Theory for smooth surfaces}

We have analyzed the adhesion data presented above using the JKR theory [34, 35]. The JKR theory is valid for elastically 


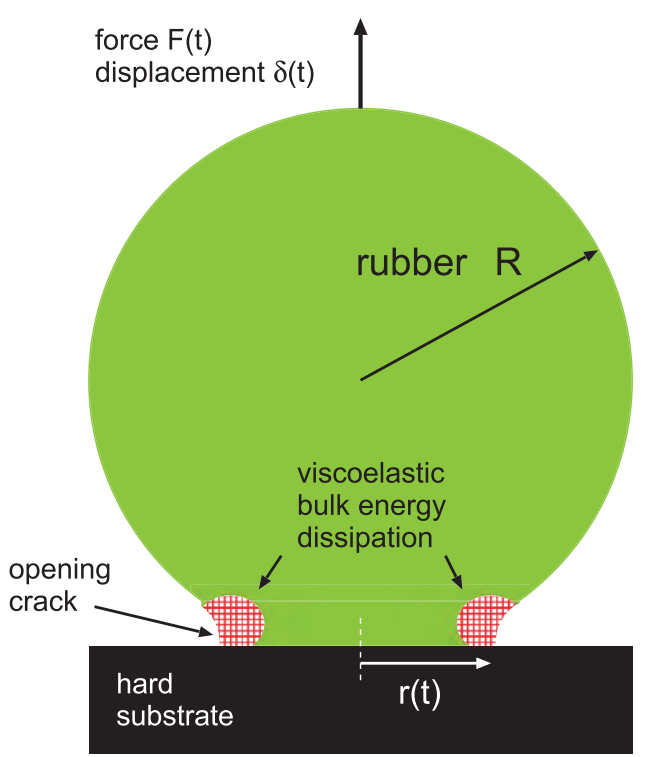

Figure 9. A rubber ball pulled off a hard flat substrate. The force necessary to separate the bodies will depend on the viscoelastic energy dissipation in the vicinity of the tip of the opening crack at $r=r(t)$.

soft solids with large enough radius of curvature ${ }^{6}$. In the JKR theory the removal of a ball from a substrate is considered as an interfacial crack propagation problem, see figure 9. The JKR approach is valid if deformations of the rubber on a length scale of the order of the radius of the contact region $r$ occur so slowly that the rubber can be considered as a perfect elastic material. This requires that $\operatorname{Im} E(\omega) / \operatorname{Re} E(\omega) \ll 1$ for the typical deformation frequencies $\omega \approx v_{z} / r$, where $v_{z}$ is the pull-off velocity. However, close to the crack tip the deformation frequencies $\omega \approx v_{\mathrm{r}} / s$, where $v_{\mathrm{r}}=-\mathrm{d} r / \mathrm{d} t$ is the crack tip velocity and $s$ the distance from the crack tip. Since $s$ may be as small as a few $\mathrm{nm}$, the deformation frequencies at the crack tip may be very high, even for a slowly moving crack, but the resulting viscoelastic energy dissipation is fully included in the crack propagation energy $\gamma_{\text {eff }}(v, T)$. We will denote $\gamma_{\text {eff }}(v, T)=\gamma_{\text {eff }}(v, T)$ as an effective interfacial energy.

If $R$ is the radius of the sphere, $r$ the radius of the sphere-substrate contact region and $\delta$ the penetration, then we have from the JKR theory:

$$
\delta=\frac{r^{2}}{R}-\left(\frac{2 \pi \gamma_{\mathrm{eff}} r}{E^{*}}\right)^{1 / 2}
$$

giving

$$
\dot{\delta}=\frac{2 r}{R}\left[1-\left(\frac{\pi \gamma_{\mathrm{eff}} R^{2}}{8 r^{3} E^{*}}\right)^{1 / 2}\right] \dot{r}
$$

6 We have calculated the Maugis or Tabor number $\lambda \approx a^{-1}\left(\Delta \gamma^{2} R / E^{2}\right)^{1 / 3}$, where $a$ is the interfacial binding distance. The $\lambda$-number is essentially the ratio of the neck height to the intermolecular spacing and the JKR theory is valid if $\lambda>5$. For van der Waals bonded systems $a \approx 0.4 \mathrm{~nm}$ and $\Delta \gamma \approx$ $0.05 \mathrm{~J} \mathrm{~m}^{-2}$, so that in our case (with $R \approx 1 \mathrm{~cm}$ and $\left.E \approx 2 \mathrm{MPa}\right) \lambda \approx 5000$, and the JKR limit prevails.

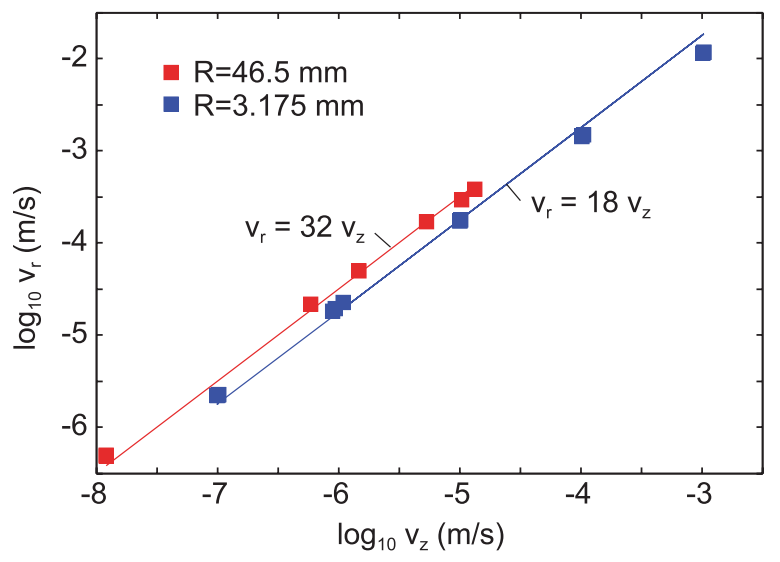

Figure 10. The calculated (using the JKR theory) crack tip velocity $v_{\mathrm{r}}$ as a function of the pull-off velocity $v_{z}$ for all experimental data.

Here we have neglected the dependency of $\gamma_{\mathrm{eff}}$ on time, which is a good approximation, at least in the present case. At the point of snap-off $r=r_{\mathrm{c}}$, where

$$
r_{\mathrm{c}}=\left(\frac{9 \pi R^{2} \gamma_{\mathrm{eff}}}{8 E^{*}}\right)^{1 / 3} .
$$

Thus, at snap-off the opening crack moves with the speed

$$
v=\dot{r}_{\mathrm{c}}=\left(\frac{3 R E^{*}}{8 \pi \gamma_{\mathrm{eff}}}\right)^{1 / 3} \dot{\delta} .
$$

The pull-off force

$$
F_{\mathrm{c}}=\frac{3 \pi}{2} \gamma_{\mathrm{eff}} R
$$

From the theory of crack propagation (section 3):

$$
\gamma_{\mathrm{eff}}=\gamma_{0}(v, T)[1+f(v, T)]
$$

where $f(v, T)$ is the viscoelastic enhancement term and $\gamma_{0}(v, T)$ the energy per unit surface area to break the bonds at the interface. This term will, in general, also depend on the crack tip velocity $v$ and the temperature $T$, but as $v \rightarrow 0$ we have $\gamma_{0} \rightarrow \Delta \gamma=\gamma_{1}+\gamma_{2}-\gamma_{12}$, where $\gamma_{1}$ and $\gamma_{2}$ are the surface energies of solid 1 and 2 , respectively, and $\gamma_{12}$ is the interfacial binding energy between solid 1 and 2. Using (6) and (7) we can also write

$$
\dot{r}_{\mathrm{c}}=\left(\frac{9 R^{2} E^{*}}{16 F_{\mathrm{c}}}\right)^{1 / 3} \dot{\delta} .
$$

To test the approach described above, we show in figure 10 the crack tip velocity $v_{\mathrm{r}}=-\mathrm{d} r / \mathrm{d} t$ as a function of the pull-off velocity $v_{z}$, as deduced from experimental data using (9) (JKR theory). For all systems with the ball radius $R=3.175 \mathrm{~mm}$ (blue squares), the ratio $v_{\mathrm{r}} / v_{z}$ at the point where the adhesion force is maximal is about 18 (blue line in figure 10). Figure 11 shows that the ratio between the measured crack tip velocity $v_{\mathrm{r}}$ and the pull-off velocity $v_{z}$, at the point where the adhesion force is maximal, is about 15 , in good agreement with the prediction of the JKR theory (see figure 10). In figure 11 the crack tip velocity has been deduced 


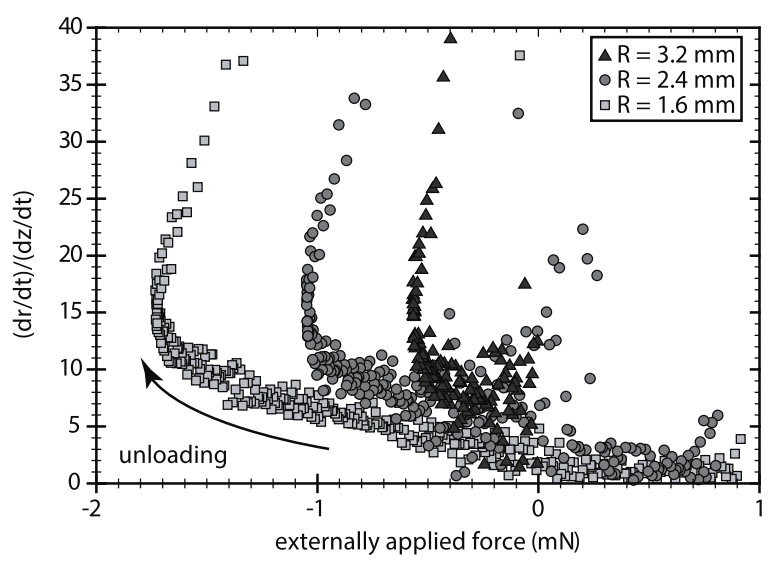

Figure 11. The ratio between the measured crack tip velocity $v_{\mathrm{r}}=|\mathrm{d} r / \mathrm{d} t|$ and the pull-off velocity $v_{z}=\mathrm{d} z / \mathrm{d} t$ is about 15 , which is in good agreement with the prediction of the JKR theory (see figure 10). Based on measurements by removing silicon nitride balls with radii $R=3.2,2.4$ and $1.6 \mathrm{~mm}$ from PDMS.

from optical inspection of how the radius of the contact region decreases with time during the removal of silicon nitride balls with radii $R=3.2,2.4$ and $1.6 \mathrm{~mm}$ from the PDMS-substrate.

\subsection{Experimental results for smooth surfaces and analysis}

We now present results for the effective interfacial crack propagation energy $\gamma_{\text {eff }}$ as obtained from the pull-off force of balls (silicon nitride or PDMS) from flat surfaces (PDMS or PMMA). We have deduced $\gamma_{\text {eff }}$ as a function of the crack tip velocity $v_{\text {crack }}=v_{\mathrm{r}}=-\mathrm{d} r / \mathrm{d} t$ using (7) and (9).

The square symbols in figure 12 show the effective interfacial energy $\gamma_{\text {eff }}$ (in units of $\gamma_{0}(0)$ ), as a function of the crack tip velocity $v_{\mathrm{r}}=-\dot{r}$, as obtained from the experimental data analyzed using the JKR theory. The used values for the crack tip bond-breaking contribution $\gamma_{0}(0)$ are $0.125 \mathrm{~J} \mathrm{~m}^{-2}$ (for red data points) and $0.046 \mathrm{~J} \mathrm{~m}^{-2}$ (for blue data points). The solid green lines are the viscoelastic factor $[1+f(v, T)]$, as a function of the crack tip velocity (from figure 8 ). In the present case the adhesion experiments were performed at room temperature $\left(T_{0} \approx(20 \pm 2)^{\circ} \mathrm{C}\right)$, so only the lowest green line is relevant, but the figure also shows that an inaccuracy $\left( \pm 2{ }^{\circ} \mathrm{C}\right)$ in the temperature would have negligible effect on the analysis ${ }^{7}$. The red squares are from the measurements performed in Jülich with an $R=46.5 \mathrm{~mm}$ PDMS ball against smooth PMMA, while the blue squares are from the University of Florida measurements with an $R \approx 3 \mathrm{~mm}$ silicon nitride ball against PDMS. Note that for $v_{\mathrm{r}}<10^{-4} \mathrm{~m} \mathrm{~s}^{-1}$ the experimental data exhibits the same velocity dependence as the calculated crack propagation factor $[1+f(v, T)]$. The velocity dependence is very weak, roughly $v_{\mathrm{r}}^{\alpha}$ with $\alpha \approx 0.12$, which reflects the very weak frequency dependence of the real part of the viscoelastic modulus (see discussion in section 3).

7 The viscoelastic enhancement factor $[1+f(v, T)]$ depends on the crack tip velocity and the temperature as the product $v a_{T}$. Thus, changing the temperature one could modify the crack propagation contribution. However, this would also change the contribution from the interfacial bond-breaking process (stress-aided, thermally activated process).

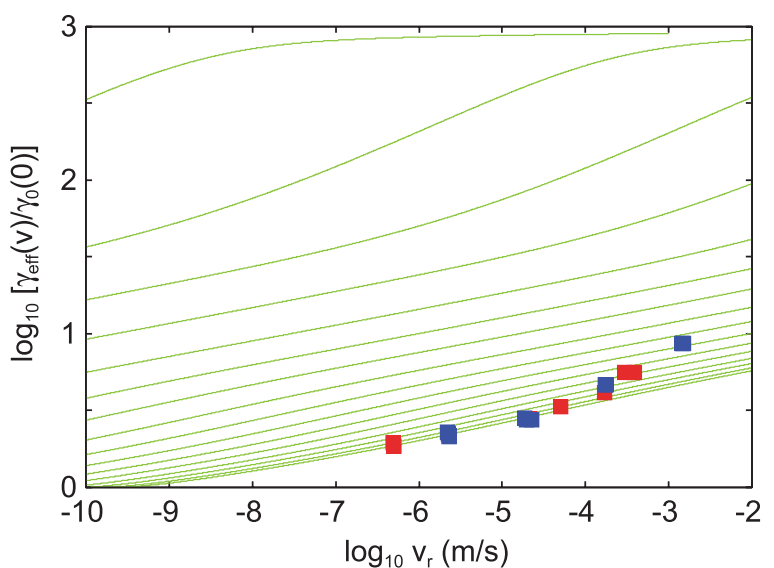

Figure 12. The square symbols are the effective interfacial energy $\gamma_{\text {eff }}(v)$ (in units of $\gamma_{0}(0)$ ), as a function of the crack tip velocity $v_{\mathrm{r}}=-\dot{r}$, obtained using the JKR theory. The red and blue squares are from the experimental data obtained in Jülich $(R=46.5 \mathrm{~mm}$ PDMS ball on PMMA) and at the University of Florida ( $R=3.175 \mathrm{~mm}$ silicon nitride ball on PDMS), with low-velocity crack tip bond-breaking contributions $\gamma_{0}(0)=0.125 \mathrm{~J} \mathrm{~m}^{-2}$ and $0.046 \mathrm{~J} \mathrm{~m}^{-2}$, respectively. The solid green lines are the viscoelastic factor $[1+f(v, T)]$ as a function of the crack tip velocity from figure 8 . The lower green curve is for the temperature $T=20^{\circ} \mathrm{C}$ of the adhesion experiments, but the figure shows that the exact value of the temperature is not very important for the viscoelastic factor $[1+f(v, T)]$ as long as it is close to $T \approx 20^{\circ} \mathrm{C}$.

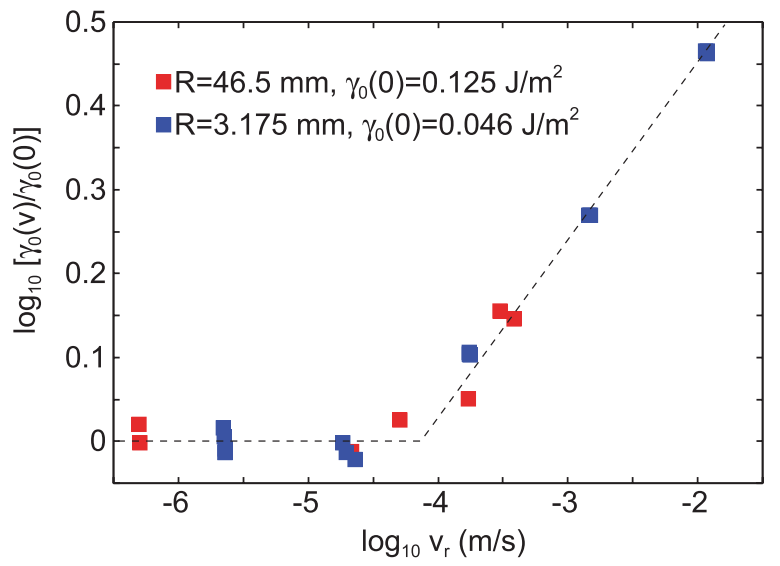

Figure 13. The square symbols are the interfacial bond-breaking contribution $\gamma_{0}(v)$ to the interfacial energy, as a function of the crack tip velocity $v_{\mathrm{r}}=-\dot{r}$, as obtained by dividing $\gamma_{\text {eff }}$ in figure 12 (squares) by the calculated viscoelastic factor $[1+f(v, T)]$ (bottom green line in figure 12). The dashed line is a fit to the data.

The square symbols in figure 13 show the interfacial bond-breaking factor $\gamma_{0}$ contribution to the interfacial energy, as a function of the crack tip velocity $v_{\mathrm{r}}=-\mathrm{d} r / \mathrm{d} t$, as obtained by dividing $\gamma_{\text {eff }}$ (from figure 12) by the calculated viscoelastic factor $[1+f(v, T)]$ (lower green line in figure 12). The dashed line is a fit to the data, where the slope of the line for $v_{\mathrm{r}}>$ $10^{-4} \mathrm{~m} \mathrm{~s}^{-1}$ corresponds to $\gamma_{0} \sim v_{\mathrm{r}}^{\mu}$ with $\mu \approx 0.21 \pm 0.01$. We interpret the velocity dependence exhibited by $\gamma_{0}(v)$ as resulting from thermally activated stress-aided processes. In particular, the removal of a chain molecule adsorbed on a 


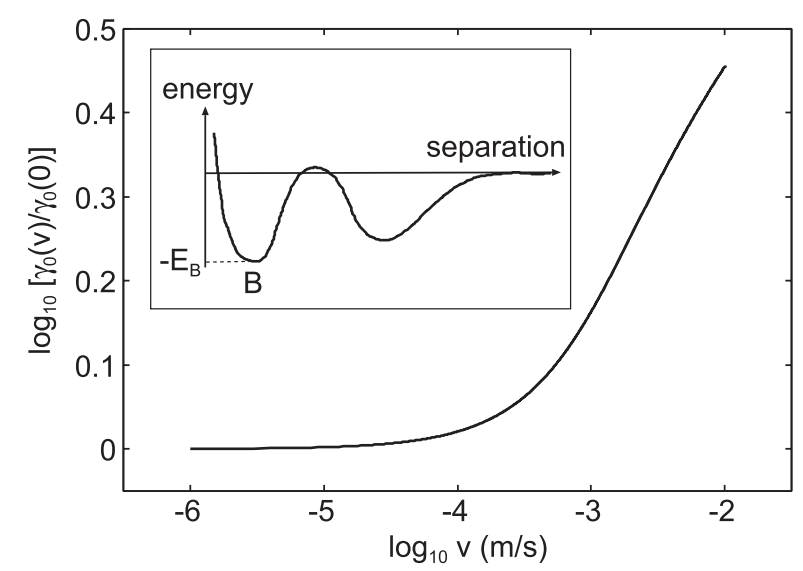

Figure 14. $\gamma_{0}(v) / \gamma_{0}(0)=1+A \sinh ^{-1}\left(v / v_{0}\right)$, as a function of the separation velocity $v=v_{\perp}$, with $A=0.7$ and $v_{0}=0.0013 \mathrm{~m} \mathrm{~s}^{-1}$.

substrate (corrugated potential) may exhibit a nearly constant pull-off energy at low velocity, which increases with velocity above some critical velocity, see [15]. The most simple model describing this effect assumes that there is an energy barrier on the separation path, as indicated by the inset in figure 14 (see section 8.3 in [1] and also [37]). Imagine first that the system is in state $B$ and we want to pull the surfaces apart. To do this we have to apply a force sufficient to overcome the energy barrier, with the help of the Brownian energy $k_{\mathrm{B}} T$. The total energy to break the bond in this case is the sum of the adiabatic work of adhesion $\gamma_{0}(0)=n E_{\mathrm{B}}$ (where $n$ is the number of adhesion units per unit surface area) and the work to overcome the energy barrier, which will depend on the separation speed $v=v_{\perp}$. This simple model predicts $\gamma_{0}(v) / \gamma_{0}(0)=$ $1+A \sinh ^{-1}\left(v / v_{0}\right)$, which is in qualitative agreement with our observations (see figure 14). Note, however, that the (vertical) separation velocity $v_{\perp}$ is related to the crack tip velocity $v_{\mathrm{r}}$ in a non-trivial way that depends on the crack tip process zone and how it is modified with increasing crack tip velocity. A more refined model would need to take into account that, in general, several barriers (and metastable states) occur on the way between the initial $E_{1}$ state and the final state $E_{2}$ (see, e.g., section 5 in [15]). See also appendix B.

\subsection{Closing crack and role of dust particles}

Viscoelastic energy dissipation in the rubber close to the opening crack tip increases the energy to propagate the opening crack with the factor $[1+f(v)]$. For a closing crack, the viscoelastic energy dissipation instead reduces the crack propagation energy, roughly by a factor $1 /[1+f(v)]$ (see $[31,36])$. Thus for a closing crack one expects $\gamma_{\text {eff }}^{\text {closing }} \approx$ $\Delta \gamma /[1+f(v)]$. Since $\Delta \gamma \approx 0.05 \mathrm{~J} \mathrm{~m}^{-2}$ and since for $v_{z} \approx$ $1 \mu \mathrm{m}$ we have $[1+f(v)] \approx 3$ we get a very small closing crack propagation energy, $\gamma_{\text {eff }}^{\text {closing }} \approx 0.015 \mathrm{~J} \mathrm{~m}^{-2}$ at typical crack tip velocities. This is in qualitative agreement with our experiments, where in the Jülich experiments almost no adhesion can be detected during the contact formation, see figure 15(a).
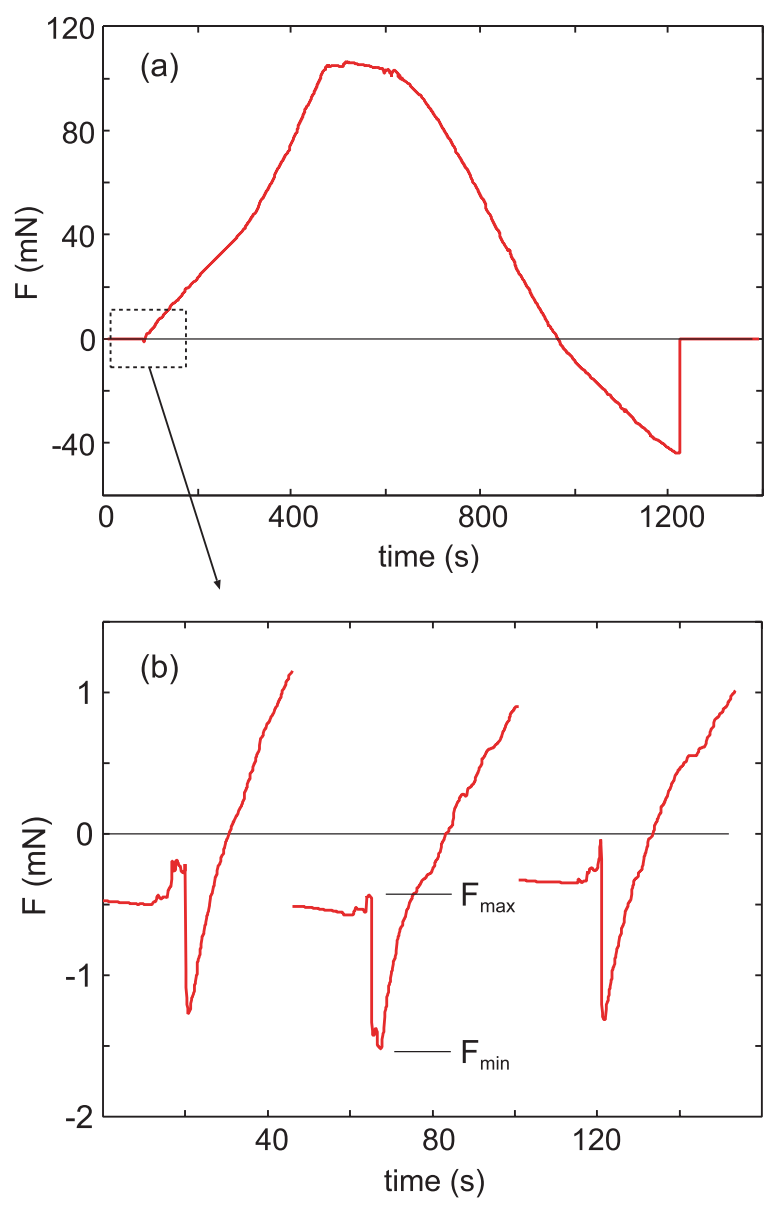

Figure 15. (a) A typical loading-unloading cycle when a PDMS sphere is brought in to and out of contact with a PMMA substrate (Jülich experiment). After contact formation the sphere is loaded until a given preload is reached, then kept for 2 min before pulling it up again at a constant velocity until the two solids are separated. In (b) we show a magnification of the point of contact formation (for three different measurements) where the maximum adhesion force $\left(F_{\min }\right)$ is observed just after a short increase in the force signal $\left(F_{\max }\right)$. The latter effect can be attributed to dust particles that are trapped in the contact zone and penetrate into the PDMS sphere during contact formation (see figure 17). The approach velocity and the separation velocity in all the experiments was $v_{z}= \pm 0.5 \mu \mathrm{m} \mathrm{s}^{-1}$.

Figure 15(b) shows on a magnified scale the force as a function of time during the contact formation, for three nominally identical adhesion tests with $v_{z}= \pm 0.5 \mu \mathrm{m} \mathrm{s}^{-1}$. Prior to contact, in all three cases there is an attractive force of the order of $\approx 0.5 \mathrm{mN}$, which is due to the electrostatic attraction between charges trapped on the PDMS ball and the PMMA substrate. A similar attraction (not shown) occurs after detachment. The maximum (attractive) adhesion force during contact formation is $F=F_{\min } \approx-1.5 \mathrm{mN}$, which is a factor of $\sim 2$ smaller than expected theoretically. Thus in figure 16 we show the relation between the force $F$ and the penetration $\delta$ as calculated using the JKR theory with $\gamma=0.014 \mathrm{~J} \mathrm{~m}^{-2}$ as expected in the present case. This gives for $\delta=0$ (the point of contact formation) the force $F_{\min } \approx$ $-2.8 \mathrm{mN}$. We believe that the difference between the observed and calculated adhesion force is due to contamination of the 


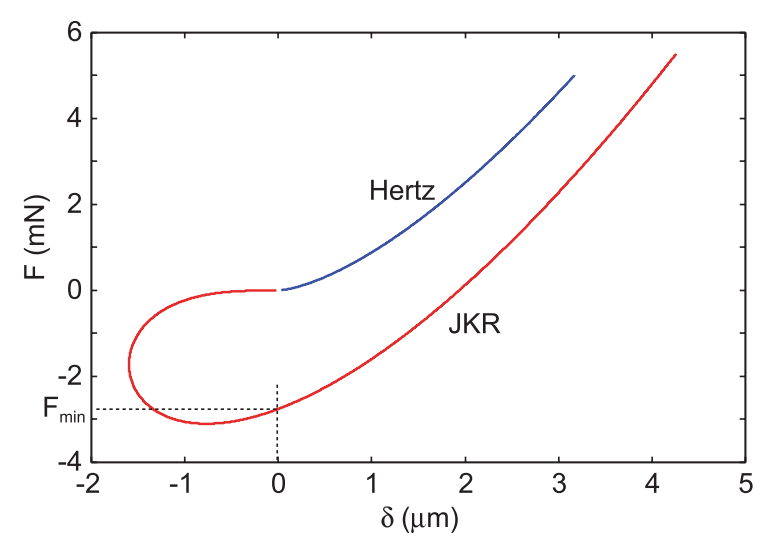

Figure 16. The relation between the penetration $\delta$ and the force $F$ for the JKR (red) and Hertz (blue) theory. In the calculation $E=2.3 \mathrm{MPa}, R=47 \mathrm{~mm}$ and $\gamma=0.014 \mathrm{~J} \mathrm{~m}^{-2}$.

surfaces with micrometer-sized particles, which cannot be avoided when working in normal atmospheric conditions. In fact, before the attractive well is observed (at $F=F_{\min }$ ), we observe an increase in the force, giving a local maximum $F_{\text {max }}$ (see figure 15(b)). We interpret this as resulting from an energetic barrier towards contact formation, induced by one or several contamination particles.

The influence of contamination particles on the adhesion is illustrated in figure 17. If a dust particle is trapped on the rubber sphere close to the lowest point on the sphere (see figure 17(a)), upon contact with the substrate the rubber must deform around the dust particle (b) until the rubber finally makes contact with the substrate (c). The elastic deformation energy in state (b) constitutes an energy barrier against contact formation, resulting in the local maximum $F_{\max }$ in the interaction force, as seen in figure 15(b). We can estimate the barrier towards contact formation using the Hertz theory. Assume that a spherical particle with radius $r_{0}$ is located on a flat rubber surface. If we apply a force $F$ to the particle it will penetrate into the rubber by a distance $\delta$ given by [35]:

$$
\delta=\left(\frac{9 F^{2}}{16 r_{0} E^{* 2}}\right)^{1 / 3} .
$$

In order for the particle to penetrate completely (as in figure 17(b)) we must have $\delta=2 r_{0}$, which gives

$$
F \approx 3.77 r_{0}^{2} E^{*} \text {. }
$$

This equation can also be derived using dimensional arguments: the work $F \delta$ must equal the elastic deformation energy. The latter is the product of the volume $\sim r_{0}^{3}$ where the deformation occurs, times the deformation energy per unit volume, which equals $\sim \sigma \epsilon \sim E \epsilon^{2}$, where $\sigma$ is the stress and $\epsilon$ the strain. With the strain $\epsilon \sim \delta / r_{0}$ we obtain the same result as above, except for a numerical factor of the order of unity. With $r_{0} \approx 5 \mu \mathrm{m}$ the equation above gives $F \approx 0.2 \mathrm{mN}$, which is similar to the height of the peak we observe before the adhesion well. Another qualitative observation in the Florida lab, supporting the picture presented above, was the absence of adhesion between a rubber ball and a substrate after the ball was kept for several months in a laboratory environment. After (a) $\mathrm{F}=0$

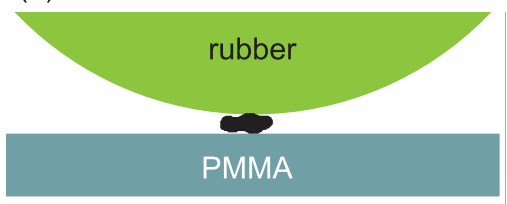

(b) $F=F_{\max }$

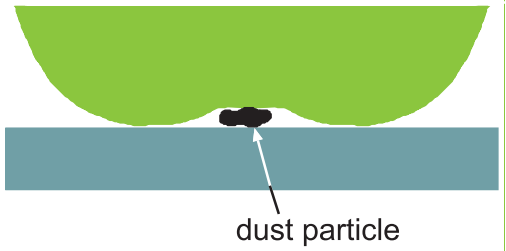

(c) $\mathrm{F}=\mathrm{F}_{\min }$

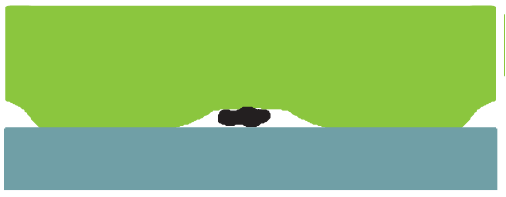

Figure 17. If a dust particle is trapped on the rubber sphere close to the lowest point on the sphere (a), upon contact with the substrate the rubber must deform around the dust particle (b) until the rubber finally makes contact with the substrate (c). The elastic deformation energy in state (b) constitutes an energy barrier against contact formation, resulting in the local maximum $F_{\max }$ in the interaction force seen in figure 15(b).

cleaning the surfaces the adhesion returned to its original value $^{8}$

We have studied a PDMS-glass interface using an optical microscope and detected several particles with a diameter of the order of $\sim 10 \mu \mathrm{m}$. We first cleaned the surfaces in a distilled water-soap mixture for $\sim 15 \mathrm{~min}$, and then rinsed the surfaces with distilled water to get rid of the soap. The surfaces were dried in a normal atmosphere. The rubber-glass interfaces were imaged at room temperature using an Axiovert 200 (Carl Zeiss Microimaging GmbH, Jena, Germany). The microscope was equipped with a LD Plan Neofluar $40 \times / 0.6 \mathrm{Ph} 2$ (Carl Zeiss) objective. The images were converted into 8-bit grayscale images and the background image was subtracted (figures 18 and 19) from the original image. For better visualization the images were inverted, the brightness and contrast adjusted and the scale bar added.

Figures 18 and 19 show two optical images with particularly large trapped particles. Note that the rubber bends around the particles and makes contact with the PMMA surface a distance away from the particle of the order of the

8 It is clear that there must be a barrier towards adhesion resulting from contamination particles, and the barrier we estimate theoretically is consistent with what we observe in the experiments. However, there could also be a very small effect resulting from charge neutralization effects in (or very close to) the PDMS-substrate contact region. However, the surface of the contact region is less than $1 / 1000$ of the total surface area of the ball and we expect a very small contribution to the change in the force from this effect, owing to the expected low surface diffusion for charged groups on the PDMS surface. 


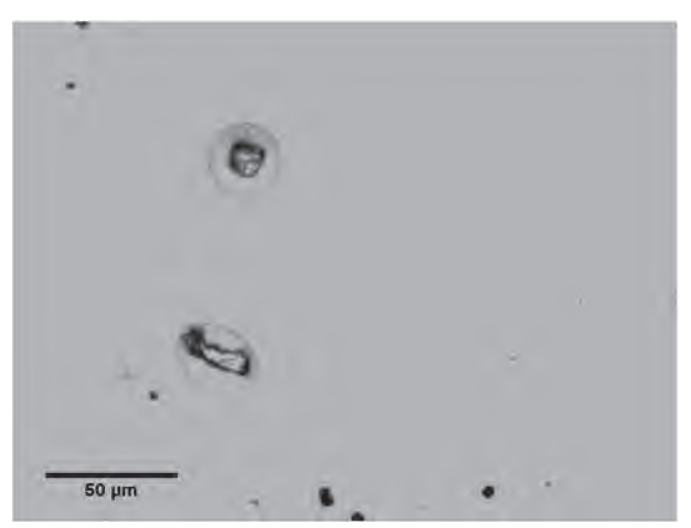

Figure 18. Optical image of the PDMS-glass interface with trapped dust particles. Note that the rubber bends around the particles and makes contact with the glass surface a distance away from the particle of the order the particle radius, giving rise to the circular rings around the particles.

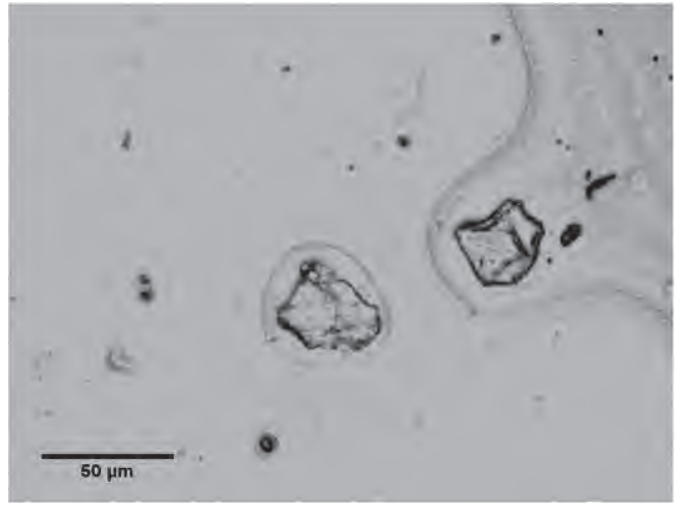

Figure 19. Optical image of the PDMS-glass interface close to the boundary line of the contact region. Note that the boundary line (crack tip) bends around a particle at the edge of the contact region.

particle radius, giving rise to the circular rings around the particles. In figure 19 it is interesting to note that the boundary line (crack tip) of the rubber-substrate contact region bends around a particle at the edge of the contact region.

For a spherical 'contamination' particle, e.g., a small glass sphere, from the radius of the particle and the radius of the circular rubber-substrate separation line (crack tip) one can calculate or estimate the interfacial binding energy $\gamma_{\text {eff }}$.

Inspection of the PDMS-glass contact region shows that, on average, several hundred micrometers separate micrometer-sized trapped particles. In the Jülich experiments the radius of the contact region is of the order of millimeters, and, on average, several micrometer-sized particles occur in the nominal contact region. In the Florida experiments the ball radius is much smaller, and the radius of the nominal contact region is of the order of $100 \mu \mathrm{m}$, i.e., the contact area is $\sim 100$ times smaller than in the Jülich experiments. Thus, in this case, and in most other adhesion experiments presented in the literature, usually no micrometer-sized particles occur in the contact region, as is also seen clearly in the optical images in figure 6(b).
Most of the particles that are observed after separating the PDMS-glass contact are adsorbed on the PDMS surface. This is expected because the dust particles have rough surfaces and will adsorb more strongly on elastically soft materials, where a larger contact area can form without storing up a lot elastic deformation energy (see section 4.4) [38].

We have performed closing crack experiments at two different velocities $v_{z}=0.5$ and $5 \mu \mathrm{m}$. On average we find that the closing crack adhesion $F_{\min }$ is about $\sim 35 \%$ larger at the lower velocity. This agrees with the theory above, since the ratio in the viscoelastic factor $\sim 1 /(1+f(v))$ for $v_{z}=0.5$ and $5 \mu \mathrm{m}$ is $\approx 1.3$, i.e. about $\sim 30 \%$ larger at the lower velocity.

\subsection{Theory for rough surfaces}

We assume that the pull-off or maximum adhesion force on all the surfaces (smooth and rough) is given by the JKR expression, $3 \pi \gamma_{\mathrm{eff}} R / 2$, and is hence proportional to the effective opening crack propagation energy (per unit surface area) $\gamma_{\text {eff }}(v)$. Here we will discuss how surface roughness affects $\gamma_{\text {eff }}(v)$, which we now denote by $\gamma_{\text {eff }}^{\text {rough }}(v)$. Let us first assume that no adhesion hysteresis occurs (i.e., the opening and closing crack propagation energies are the same), and that the solids are purely elastic (no viscoelasticity). For this case we predict that, contrary to our experimental observations (see section 4.5), the pull-off force should vanish for all the rough surfaces 1, 2 and 3 used in the present study. The origin of this effect is that the elastic energy stored at the interface in the vicinity of the asperity contact regions is given back during pull-off and helps to break the interfacial bonds between the rubber and the PMMA substrates. We now discuss this effect in detail.

In the theory developed in $[39,40]$ one of us has studied the dependence of the area of contact $A(\zeta)$ and the interfacial energy (per unit surface area) $\gamma(\zeta)$ on the magnification $\zeta$. When we observe the contact at a magnification $\zeta$ it is assumed that no surface roughness with wavevectors $q>\zeta q_{0}$ exists, i.e., the surfaces appear smooth at length scales shorter than $\lambda=2 \pi / q=2 \pi /\left(q_{0} \zeta\right)$. As the magnification increases, more roughness is observed and the area of (apparent) contact decreases. The nominal or apparent contact area $A_{0}=A(1)$ is the contact area observed at the lowest magnification, where the surfaces appear smooth. The true (atomistic) contact area $A_{1}=A\left(\zeta_{1}\right)$ is the contact area observed at the highest magnification $\zeta_{1}$, where all the surface roughness components are taken into account. $\gamma(\zeta)$ is the interfacial binding energy (per unit area) in the contact area $A(\zeta)$ when the interface is studied at a magnification $\zeta$. It follows that $\gamma\left(\zeta_{1}\right)=\Delta \gamma=$ $\gamma_{1}+\gamma_{2}-\gamma_{12}$, while $\gamma(1)=\gamma_{\mathrm{eff}}^{\text {rough }}$ is the effective macroscopic interfacial energy in the surface area $A_{0}=A(1)$, which is the quantity which enters in the JKR theory. Thus, if $\gamma(1)=0$ the pull-off force will vanish, i.e., no adhesion will manifest itself at the macroscopic scale. In the theory developed in [39] (see also appendix $\mathrm{C})$ :

$$
\gamma(1) A_{0}=\Delta \gamma A\left(\zeta_{1}\right)-U_{\mathrm{el}}
$$

where $U_{\mathrm{el}}$ is the elastic energy (stored at the interface) due to the deformations of the solids necessary in order to 


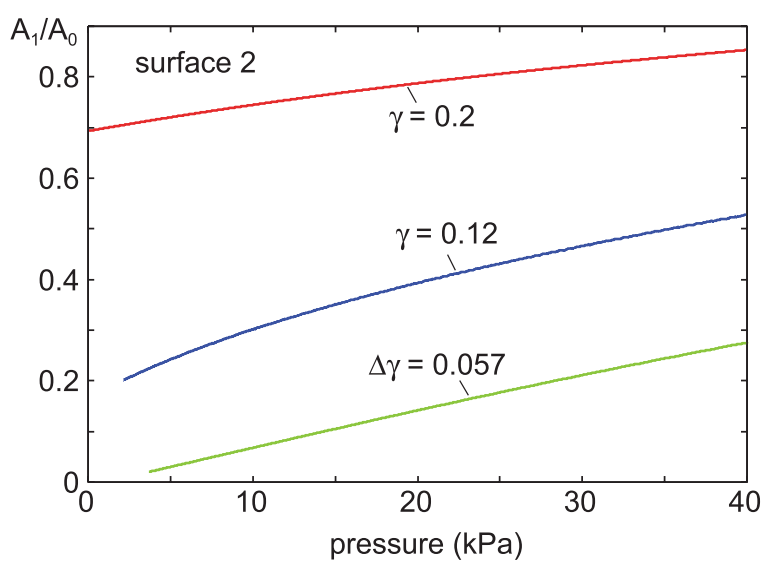

Figure 20. The relative area of contact, $A\left(\zeta_{1}\right) / A_{0}$, for PDMS against the rough PMMA surface 2 , as a function of the squeezing pressure, for the interfacial binding energies $\gamma=0.2,0.12$ and $0.057 \mathrm{~J} \mathrm{~m}^{-2}$. The figure shows that if $\gamma=\Delta \gamma=0.057 \mathrm{~J} \mathrm{~m}^{-2}$ during loading (closing crack) then the contact area formed at $p=40 \mathrm{kPa}$ will not change until $p \approx 8 \mathrm{kPa}$ if during unloading $\gamma=0.12 \mathrm{~J} \mathrm{~m}^{-2}$, while if $\gamma=0.2 \mathrm{~J} \mathrm{~m}^{-2}$ a large negative pressure would be necessary before the contact area formed during the loading act would start to decrease. Such a large negative pressure prevails in the (JKR) ball-substrate contact only close to the rim (opening crack) of the contact, see figure 23.

make contact at the interface. In the present application, if there were no contact hysteresis, and if $\Delta \gamma \approx 0.057 \mathrm{~J} \mathrm{~m}^{-2}$ (as expected for the PDMS-PMMA interface at thermal equilibrium), then the theory above predicts that the pull-off force vanishes for all the rough surfaces 1,2 and 3 used in the present study. This is illustrated by the green curve in figure 20, which shows the calculated relative area of contact, $A\left(\zeta_{1}\right) / A_{0}$, for PDMS against the rough PMMA surface 2, as a function of the squeezing pressure. Note that as the pressure approaches zero, the contact area vanishes and so will the pull-off force. However, the interfacial adhesion does increase the contact area when the load (or squeezing pressure) is non-vanishing. This is illustrated in figure 21 , which shows the variation of the relative area of contact, $A(\zeta) / A_{0}$, and the relative effective interfacial energy, $\gamma(\zeta) / \Delta \gamma$, with the logarithm of the magnification $\zeta=q / q_{0}$ for PDMS rubber squeezed against the rough PMMA surfaces 1, 2 and 3 . The normal pressure $p=40 \mathrm{kPa}$, which is the typical pressure acting in the central part of the contact region when the load is $0.1 \mathrm{~N}$ (see figure 23). Note that, for magnifications larger than $\zeta_{\mathrm{c}} \approx 100$, in all cases the contact area becomes independent of the magnification, i.e., the surfaces are in complete contact within the asperity contact regions which can be observed at a magnification $\zeta_{c}$. In the absence of adhesion the contact area would instead continue to decrease as the magnification increases beyond $\zeta_{\mathrm{c}}$, as a new shorter wavelength roughness now would be observed.

Note that in all cases the effective interfacial energy vanishes before reaching a magnification $\zeta=1$. Thus, as stated above, if there were no adhesion hysteresis (i.e. the effective interfacial energy for the opening and closing crack is equal) then the pull-off force would vanish for all the rough PMMA substrate surfaces. Let us now include the adhesion

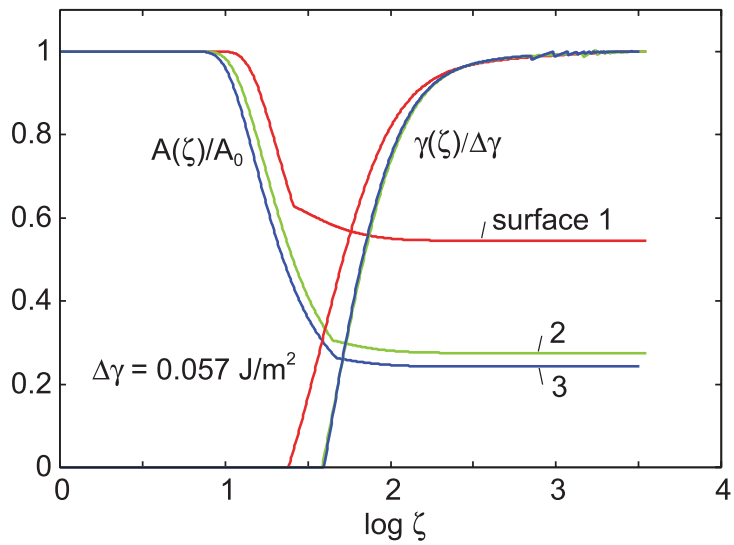

Figure 21. The variation of the relative area of contact, $A(\zeta) / A_{0}$, and the relative effective interfacial energy, $\gamma(\zeta) / \Delta \gamma$, with the logarithm of the magnification $\zeta=q / q_{0}$ for PDMS rubber squeezed against the rough PMMA surfaces 1,2 and 3. The normal pressure $p=40 \mathrm{kPa}$ and the interfacial energy $\Delta \gamma=0.057 \mathrm{~J} \mathrm{~m}^{-2}$.

hysteresis. We will show that in this case a large negative pressure (as prevails only close to the edges of the (JKR) macroscopic contact region during pull-off) is necessary in order to break the asperity contact regions. That is, the elastic energy stored in the vicinity of the asperity contact regions, which determines the asperity contact strain energy release rate $G$, is not large enough (i.e. $G<\gamma_{\text {eff }}$ ) to propagate an opening crack (and reduce the size of the asperity contact region), unless the asperity contact is in the macroscopic crack tip process zone.

Assume that at the end of the loading cycle the asperity contact regions appear as shown in figure 22(a). In the absence of adhesion hysteresis (figure 22(b)), during unloading the asperity contact regions would disappear in a similar way as they were formed, and asperity contact regions even at the center of the macroscopic contact area will decrease in size even at the start of unloading, where the radius $r(t)$ of the macroscopic separation line (dashed lines in figure 22) is far from the center of the contact region. However, if the adhesion hysteresis is large enough so that the energy per unit area for the opening crack $\gamma_{\text {eff }}(v) \gg \Delta \gamma$, then the asperity contact regions will only start to shrink when they are very close to the macroscopic opening crack (in the crack tip process zone) (figure 22(c)). In this case the pull-off force will be non-vanishing, and to a good approximation given by the JKR theory with $\gamma_{\text {eff }}^{\text {rough }} \approx \gamma_{\text {eff }}(v) A_{1} / A_{0}$, where $\gamma_{\text {eff }}(v)$ is the effective interfacial energy obtained from the contact between smooth surfaces (shown by the squares in figure 12). This is illustrated in figure 22(c), where the size of the asperity contact regions outside of the crack tip process zone remain unchanged (in spite of the reduction in the contact pressure). Very close to the tip of the macroscopic (apparent) opening crack (dashed circle) the asperity contact regions are broken by the propagation of microscopic opening cracks at each asperity contact region. In this case, as stated above, $\gamma_{\mathrm{eff}}^{\text {rough }} \approx$ $\gamma_{\text {eff }}(v) A_{1} / A_{0}$.

We now show that in the present case the adhesion hysteresis is strong enough so that the asperity contact regions 
(a)

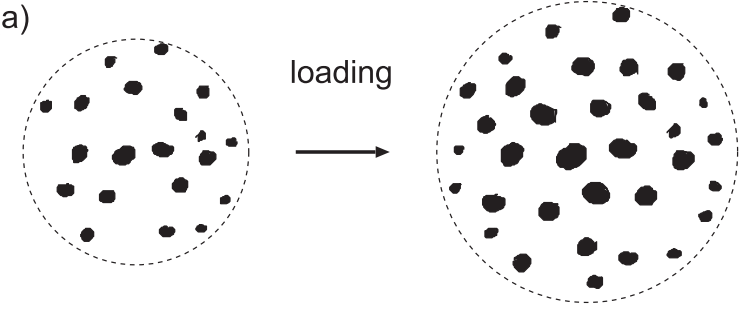

(b)

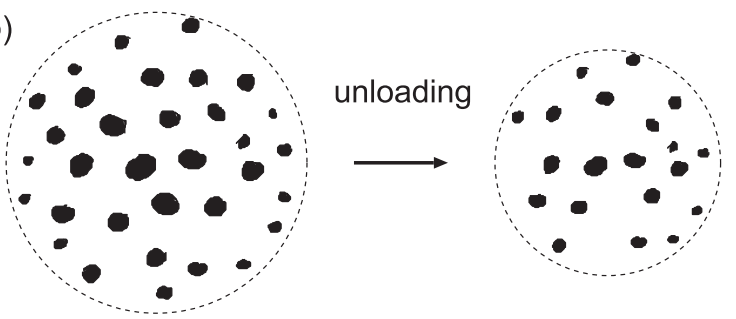

(c)

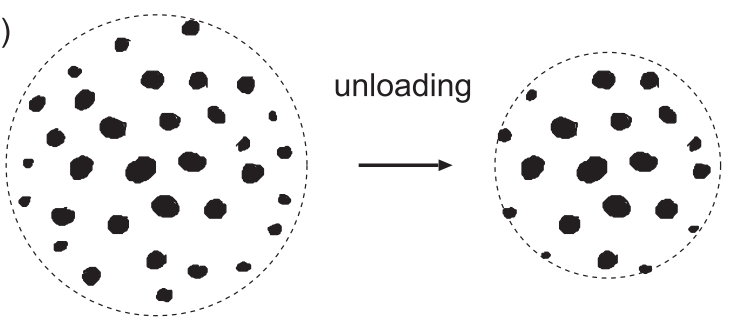

Figure 22. Schematic picture of the macroscopic contact area (a) during loading and (b) during unloading in the absence of adhesion hysteresis, and (c) when strong adhesion hysteresis occurs so that $\gamma_{\text {eff }} \gg \Delta \gamma$. The black regions indicate asperity contact regions. Because of the adhesive interaction, complete contact occurs within the black regions. During pull-off in case (b), the asperity contact regions decrease in size everywhere. During pull-off in case (c), the size of the asperity contact regions remain unchanged (in spite of the reduction in the contact pressure) except close to the macroscopic (apparent) opening crack tip (dashed circle) where the asperity contact regions are broken by the propagation of microscopic opening cracks at each asperity contact region.

are broken only close to the macroscopic separation line (tip of opening crack) ${ }^{9}$. Figure 20 shows, for PDMS against the rough PMMA surface 2 , the relative area of contact as a function of the squeezing pressure, for the interfacial binding energies $\gamma=0.2,0.12$ and $0.057 \mathrm{~J} \mathrm{~m}^{-2}$. The opening crack propagation energy is of the order of $0.2 \mathrm{~J} \mathrm{~m}^{-2}$, even at the lowest propagation velocity studied in our experiments (see figure 12). Figure 20 shows that in this case a strongly negative pressure is necessary before $A_{1} / A_{0} \approx 0.2$, which is the relative contact area resulting from the loading act

9 Adhesion hysteresis is practically always observed in reality, e.g., the viscoelastic contribution enhances the effective interfacial energy during pull-off (opening crack) and reduces it during approach (closing crack). But also the interfacial bond formation and bond breaking is almost always hysteretic, where usually elastic instabilities occur during bond breaking (snapping processes) which dissipate energy and increase the effective interfacial energy during the pull-off, in particular if long chain molecules are involved. More complex processes such as interdiffusion, which would depend on the time of stationary contact (before the separation process starts), may also occur and increase the effective interfacial energy relevant for the separation. In general, only if the approach and separation velocities were extremely slow would one expect no hysteresis in the interfacial energy, but this adiabatic limit may be impossible to reach in many cases.

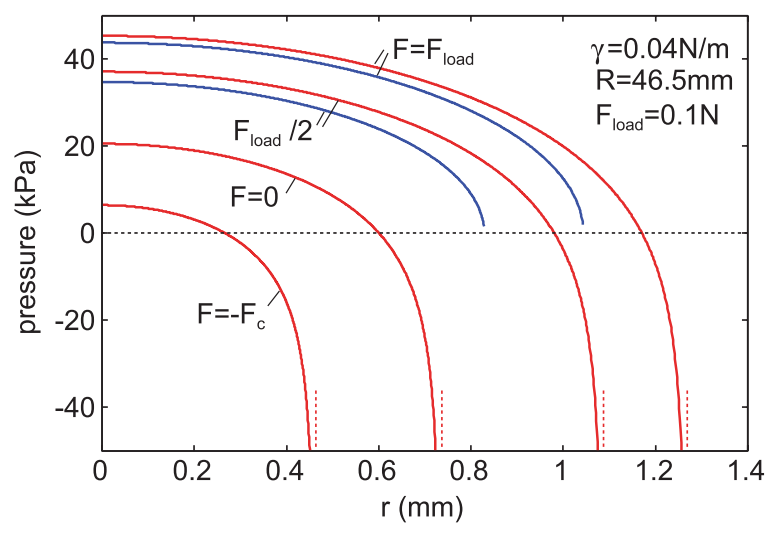

Figure 23. The contact pressure as a function of the radial position for a PDMS ball at different normal forces. $F_{\mathrm{c}}=3 \pi \gamma R / 2$ is the maximum JKR adhesion force. The red lines are the JKR theory predictions and the blue lines are the Hertz theory predictions. In the JKR theory we have used $\gamma=0.04 \mathrm{~J} \mathrm{~m}^{-2}$, which is roughly the observed crack-opening energy for PDMS against the rough PMMA surface 2 or 3 .

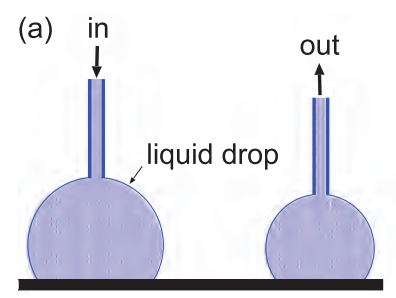

no contact angle hysteresis

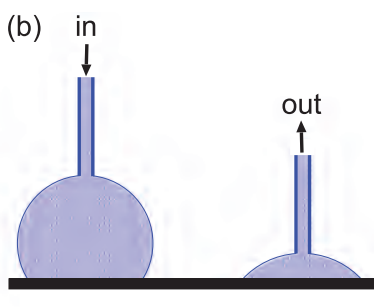

contact angle hysteresis
Figure 24. A liquid droplet on a substrate. (a) In the absence of contact angle hysteresis, when the fluid is injected or redrawn from the droplet, the size of the fluid contact region changes in such a way that the shape of the droplet (spherical cup) is unchanged. (b) When contact angle hysteresis occurs, the size of the liquid-solid contact region does not change until enough fluid has been pulled away from the droplet such that the contact angle is reduced to a lower critical value.

(green line in figure 20). A high negative pressure prevails only close to the macroscopic opening crack tip, so only here will the asperity contact regions decrease in size and (finally) break. This is illustrated in figure 23, which shows the contact pressure as a function of the radial position for a PDMS ball at different normal forces. Here $F_{\mathrm{c}}=3 \pi \gamma R / 2$ is the maximum JKR adhesion force.

The adhesion hysteresis effect illustrated in figure 22 is very similar to liquid contact angle hysteresis, see figure 24 . In the latter case there is hysteresis in the fluid-substrate contact area as a function of the fluid (Laplace) pressure in the droplet.

To summarize, for the surfaces 1-3 studied, our contact mechanics theory predicts that at equilibrium no adhesion would occur with the PDMS ball. Nevertheless, we observe a non-zero pull-off force (see below) after first bringing together the solids with a squeezing force in the range $0.1-0.2 \mathrm{~N}$. We interpret this as a kinetic effect: during separation at finite velocity there is not enough time for the equilibrium state to develop, as clearly seen in figure 12. As shown below, the experimental data can be explained if it is assumed that the 


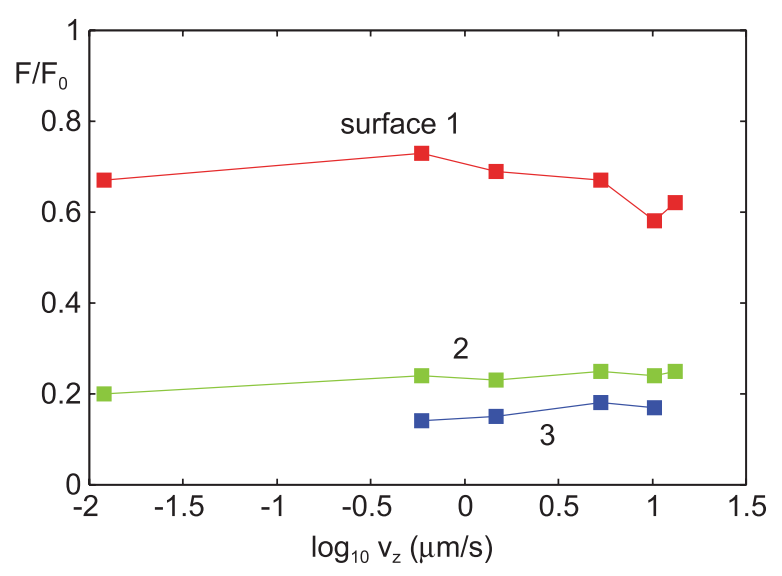

Figure 25. The ratio $F / F_{0}$ between the pull-off force $F$ on the rough surfaces 1, 2 and 3 , and the pull-off force $F_{0}$ on the smooth surface, as a function of the pull-off velocity.

energy per unit area to propagate the interfacial opening crack is given by $\gamma_{\mathrm{eff}}^{\text {rough }} \approx \gamma_{\mathrm{eff}}(v) A_{1} / A_{0}$.

\subsection{Experimental results for rough surfaces and analysis}

We have measured the pull-off force $F$ for both smooth and rough PMMA surfaces with the surface roughness power spectra shown in figure 3. In the analysis we assume that the sandblasting does not change the surface chemistry but only the surface topography ${ }^{10}$. Figure 25 shows the ratio $F / F_{0}$ between the pull-off force $F$ on the rough surfaces 1 , 2 and 3 , and the pull-off force $F_{0}$ on the smooth surface, as a function of the pull-off velocity. In all cases the maximum load before pull-off was $\approx 0.1 \mathrm{~N}$. When the velocity increases from 0.012 to $13.28 \mu \mathrm{m} \mathrm{s}^{-1}$, the pull-off force for the flat surface increases by about a factor of three (from about 0.051 to $0.154 \mathrm{~N}$ ). However the change in the ratio $F / F_{0}$ in this velocity range is very small, i.e., the pull-off force on the rough surfaces exhibits the same velocity dependence as on the smooth surfaces. The average ratios $\left\langle F / F_{0}\right\rangle$ (over all the data points) for the surfaces 1,2 and 3 are $0.66,0.235$ and 0.16 , respectively.

We can explain the reduction in the pull-off force between the smooth and rough surfaces by assuming that the pull-off force is proportional to the relative contact area $A_{1} / A_{0}$ (see section 4.4 and [41]) at maximal load. Thus we assume $\gamma_{\mathrm{eff}}^{\text {rough }}(v)=\gamma_{\mathrm{eff}}(v) A_{1} / A_{0}$, where $\gamma_{\mathrm{eff}}(v)$ is the energy to propagate the interfacial opening crack on the smooth

\footnotetext{
${ }^{10}$ The adhesion depends sensitively on the last monolayer (e.g., contamination layer) of molecules on the solid surfaces and we cannot exclude that the sandblasting may have modified the surface energy of the substrate. However, if such a modification were to occur, e.g., due to removal of a thin surface layer during the sandblasting process, one would expect similar changes to occur for all three sandblasted surfaces, whereas we observe a strong drop in the adhesion with increasing sandblasting time (compare surface 1 with surface 2 and 3 in figure 25), which also agrees with what we predict theoretically. In addition, if the sandblasting were to affect the interfacial energy one would expect (if anything) it to increase the interfacial energy by, e.g., inducing bond breaking or oxidation of the top surface layer, or removal of contamination layer, whereas we observe a strong reduction in the adhesion.
}

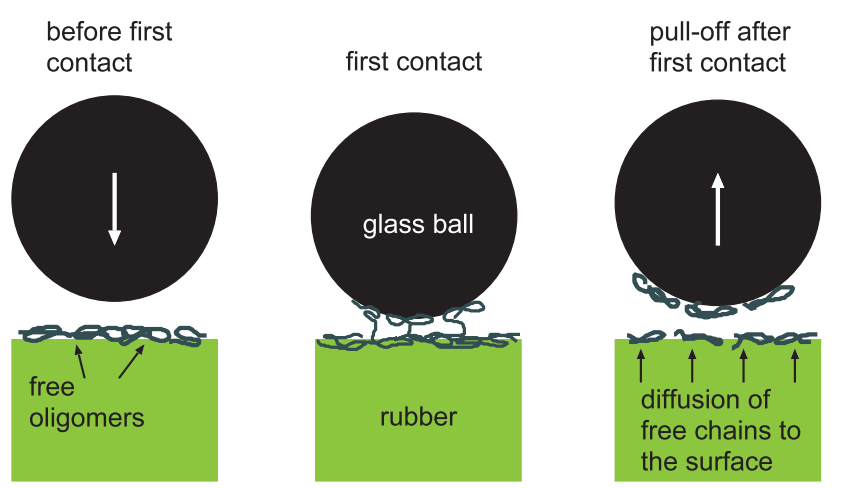

Figure 26. Mechanism for the decrease in pull-off force upon repeated contact. The transfer of oligomers between the sample (PDMS rubber) and probe (glass sphere) results in a pull-off force which changes after every new contact until a steady state configuration has been obtained. The study in [42] shows that 1000 contacts (or more) may be necessary in order to obtain the steady state.

surface. We calculate $A_{1} / A_{0}$ using the contact mechanics theory of Persson and include adhesion with the interfacial binding energy $\gamma=0.057 \mathrm{~J} \mathrm{~m}^{-2}$ (closing crack). As shown in figure 21 (for $\zeta>\zeta_{\mathrm{c}} \approx 100$ ), this gives the relative contact area $A_{1} / A_{0}=0.55,0.28$ and 0.24 for surfaces 1,2 and 3 , in relative good agreement with the ratio $\left\langle F / F_{0}\right\rangle$ for the surfaces 1,2 and $3(0.66,0.235$ and 0.16 , respectively).

As discussed in section 4.4, the basic assumption behind our approach is that the asperity contact regions, which are formed during the loading act, are broken (or reduced in size) only very close to the rim of the apparent contact region (in the crack tip process zone). If the radial width $\Delta r$ of this region is much smaller than the radius $r(t)$ of the (apparent) contact region, the JKR theory can be used to analyze the pull-off experiments.

\section{Discussion}

The experimental results presented in section 4.2 were obtained using two different experimental setups, and give different magnitudes of the effective interfacial energy $\gamma_{\text {eff }}$. In particular, $\gamma_{0}=0.046$ and $0.125 \mathrm{~J} \mathrm{~m}^{-2}$ for the experiments with a small silicon nitride ball $(R \approx 3.2 \mathrm{~mm})$ against PDMS, and for a large PDMS ball $(R \approx 47 \mathrm{~mm})$ against PMMA. We believe that this difference reflects the surface conditions of the probe and the substrate. Thus, 10:1 Sylgard PDMS, cross-linked according to the standard procedures used in our studies, has a large fraction of uncrosslinked chains, of the order of $3.6 \%$ of the mass or volume (as deduced from extraction of free chains by swelling in hexane), which can diffuse to the surface forming a thin liquid-like film. During contact with the countersurface, oligomers can be transferred to the countersurface, as illustrated in figure 26 , which will modify the interfacial interaction energy and the pull-off force. This effect has been studied in great detail by Kroner et al [42]. For a glass ball (radius $R=2 \mathrm{~mm}$ ) repeatedly pushed against and removed from a PDMS surface (pull-off velocity $v_{z}=2 \mu \mathrm{m} \mathrm{s}^{-1}$ ), they found in one case that the 


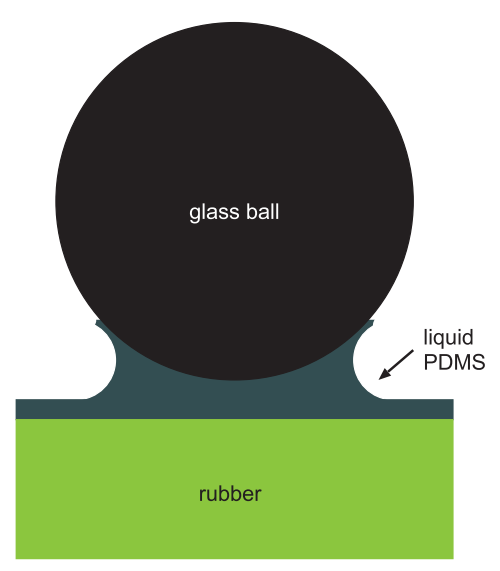

Figure 27. After very long contact time, or after repeating contact many times, a capillary bridge of oligomers may form between the sample (PDMS rubber) and the probe (glass ball). In this case, when the thickness of the capillary bridge is large enough for continuum mechanics to be valid, the work of adhesion at slow separation would be twice the surface tension of liquid PDMS, i.e. about $0.04 \mathrm{~J} \mathrm{~m}^{-2}$.

pull-off force dropped from $\approx 0.0034 \mathrm{~N}$ at first contact to $\approx 0.0012 \mathrm{~N}$ after 1000 contacts (see figures 2(b) in [42]). Using the theory of section 4.1 , this corresponds to $\gamma_{\text {eff }}=$ $0.36 \mathrm{~J} \mathrm{~m}^{-2}$ and $0.13 \mathrm{~J} \mathrm{~m}^{-2}$, respectively, and, after removing the viscoelastic factor $[1+f(v)]$, to $\gamma_{0}=0.12 \mathrm{~J} \mathrm{~m}^{-2}$ and $0.042 \mathrm{~J} \mathrm{~m}^{-2}$, respectively. The latter value corresponds to about twice the surface tension of silicone oil (the free chain component of PDMS rubber), which would be the $\gamma_{0}$ expected if adhesion were to result, e.g., from a fluid capillary bridge, as indicated in figure 27 . These values are also similar to those obtained in section 4.2 .

In [43] it was observed that when a PDMS ball was removed from a flat PDMS surface the work of adhesion $\gamma_{\text {eff }}=0.056 \mathrm{~J} \mathrm{~m}^{-2}$, while when the free chains were extracted $\gamma_{\text {eff }}=0.33 \mathrm{~J} \mathrm{~m}^{-2}$. This was tentatively attributed to chemical effects and the formation of hydrogen bonds across the interface, which is known to happen if the PDMS surface is oxidized (e.g., exposed to oxygen plasma) [44]. However, the study above and in [42] indicate that the explanation may be related to the involvement of free chains. In this context we note that we have also observed stronger adhesion for PDMS with extracted chains. We conclude that Sylgard PDMS is not such an ideal elastic rubber as usually assumed, but exhibits a large $\tan \delta$ (i.e., large dissipation) already for small perturbing frequencies (see figure 1), and has very complex surface properties.

In [16] the pull-off force was studied between a PDMS rubber ball (the radius $R=4.233 \mathrm{~mm}$ ) and seven different surfaces made from epoxy resin and produced from templates of evaporated aluminum films. These surfaces have rms roughness values much smaller than those used in the present study, namely 0.03 to $0.2 \mu \mathrm{m}$, as compared to surfaces $1-3$ with $h_{\mathrm{rms}}=1.49,2.09,2.58 \mu \mathrm{m}$. In figure 28 we compare the surface roughness power spectra of surfaces 1-3 with surface 6 used in [16], which has an rms roughness about $0.165 \mu \mathrm{m}$. The surfaces with rms roughness from $0.12 \mu \mathrm{m}$ to $0.2 \mu \mathrm{m}$ in [16] all exhibited a similar reduction in the pull-off force

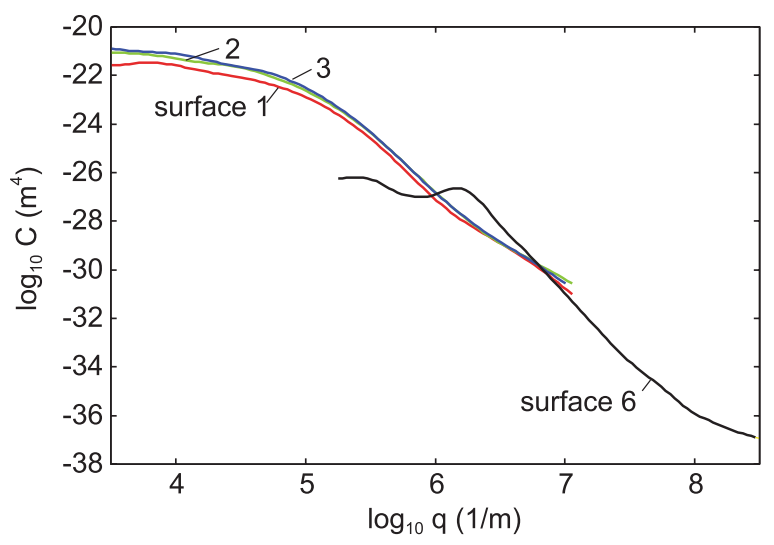

Figure 28. The surface roughness power spectra of the surfaces 1, 2 and 3 used in the present study and of surface 6 used in [16] as a function of the wavevector $\left(\log _{10}-\log _{10}\right.$ scale).

(as compared to a flat surface) to surfaces 2 and 3 in the present study, and in particular the pull-off force on surface 6 was $F / F_{0} \approx 0.2$. However, the contact mechanics seems to be very different. Thus the study in [16] showed that almost complete contact occurs within the nominal contact area of surface 6 , and the reduction in the pull-off force was attributed to the reduction in the effective interfacial energy from the elastic energy $U_{\mathrm{el}}$ stored at the interface and resulting from the bending of the bottom surface of rubber which is necessary in order for contact to occur. In the present study, for surfaces 2 and 3 , we instead found (at the load $\sim 0.1 \mathrm{~N}$ ) the contact area to be only a fraction $\sim 0.2$ of the nominal contact area, which was also the reduction found in the pull-off force. We have shown above that in the present experiments the elastic energy $U_{\mathrm{el}}$ has only a small effect on the energy necessary to break the adhesive bonds at the interface, and that the observed non-vanishing pull-off force is mainly due to the strong adhesion hysteresis.

In a sequence of papers, Kröger et al [45-47] have studied the adhesion between rubber and smooth and rough surfaces. In the experiments, a rubber ball is kept in contact with a substrate for a given time period, and then removed rapidly, typically on a few milliseconds time scale, from the substrate. The experiments show relative strong adhesion even to a substrate as rough as corundum paper or asphalt road surfaces. No detailed analysis of the experimental data is possible, as the surface topography and rubber viscoelastic properties were not studied, but the observed pull-off force is consistent with the JKR type of analysis presented above, with $\gamma_{\mathrm{eff}}^{\text {rough }} \approx \gamma_{\mathrm{eff}}(v) A_{1} / A_{0}$. In particular, Kröger et al observed that the pull-off force appears to approach zero continuously as the preload approaches zero.

\section{Summary and conclusion}

We have studied the adhesion between PDMS rubber and smooth and rough PMMA surfaces, and smooth silicon nitride surfaces. In agreement with earlier studies we have found that there are large variations in the strength of the adhesion depending both on the surface contamination or 
preparation procedure and on the number of contact cycles between the rubber and the substrate. The latter effect is most likely due to transfer of free rubber polymer chains to the countersurface. For smooth surfaces we have shown that the viscoelastic energy dissipation in the rubber at the crack tip gives an important contribution $[1+f(v)]$ to the crack propagation energy. Furthermore, as expected, the interfacial bond-breaking contribution $\gamma_{0}(v)$ also depends on the crack tip velocity $v$. We have shown that surface roughness may strongly reduce or even eliminate the adhesion between PDMS rubber balls and the countersurface. In the present study this is mainly due to the reduction in the area of real contact, and to a smaller extent due to the elastic energy stored at the contact interface, which is (partly) given back during removal of the contact.

\section{Acknowledgments}

We thank A Chateauminois for sending us their measured viscoelastic modulus of PDMS shown in figure 1 (Paris). We thank M K Chaudhury for interesting discussions about the bond-breaking contribution $\gamma_{0}(v)$ to the effective interfacial energy (section 4.2).

\section{Appendix A. Contribution from charging to the work of adhesion}

The negative force seen, e.g. in figure 5, is due to charges trapped on the PDMS and the PMMA surfaces. The mobility (in the bulk or on the surface) of charges on the PDMS and on the PMMA is likely to be very low (these materials are large-gap insulators) and there is no reason why charge neutralization should occur during the finite contact time. We note that in the Jülich experiment the rubber ball is very large (diameter almost $10 \mathrm{~cm}$ ) and it is likely that the surface charges are more or less uniformly distributed on the ball, so that very long diffusion distances would be involved for charge neutralization to occur. The charging effect seen in figure 5 is, however, exceptional large (due to the preparation of the system), and in the actual experiments the charging effect is $\approx 100$ times smaller (as in figure 15(b)), but even in these latter cases there is probably no time for charge neutralization to occur, and the attractive electrostatic force after separation is nearly as large as before contact (not shown in figure 15). However, experiments have shown that charging (typically net charge densities $\sim 1 \mathrm{nC} \mathrm{cm}^{-2}$, which is roughly $\sim 10$ times more than what we observe) always occurs when two polymer solids (even if identical materials) are separated after mechanical (adhesive or frictional) contact, see e.g., [22] (and references therein) and [48]. We expect the charging effect to depend on the external conditions, e.g., air humidity or electrically charged ions in the atmosphere, but we have not studied these (interesting) effects systematically. We estimate that in our case the electrostatic contribution to the work of adhesion during separation is completely negligible (see figure 15(a)), and has negligible influence on our measured results. (Note: we do not include in the work of adhesion the electrostatic contribution resulting from separation of the solids by a large distance, as this contribution may be non-negligible due to the long-range nature of the electrostatic contribution.) We note that in the paper by Fuller and Tabor [7], for PDMS rubber balls in contact with PMMA, they also observed charging effects, which they reduced by exposing the system to an $\alpha$-source $\left({ }^{210} \mathrm{Po}\right)$.

\section{Appendix B. On the velocity dependence of the work of adhesion}

Maugis and Barquins claim that for polyurethane the effective work of adhesion during separation scales with the crack tip velocity as $v^{0.6}$, which they interpreted as due entirely to the viscoelastic energy dissipation in the rubber in the vicinity of the crack tip. In [31] it was argued theoretically that, for high velocity, if $\operatorname{Re} E(\omega) \sim \omega^{\beta}$ then $[1+f(v, T)] \sim v^{\alpha}$, where $\alpha=\beta /(1+\beta)$. In general, $\operatorname{Re} E(\omega)$ will not be a perfect power of the frequency and $[1+f(v)]$ will not be a perfect power of the velocity, but rather a more complex function (see figure 8 for the case of PDMS). Thus the exponent $\alpha$ is not a universal number, but takes different values depending on the viscoelastic modulus; e.g., for SB rubber the measurements of Gent [30] give a region where the power law holds with $\alpha=0.27$. The exponent $\alpha$ we calculate for polyurethane using the measured viscoelastic modulus (where $\beta \approx 0.23$ in some intermediate frequency range; see, e.g., [49]) is about 0.19 , i.e. rather different from the 0.6 found for the effective interfacial energy for polyurethane by Maugis and Barquins. However, they do not take into account in the analysis that the bond-breaking contribution $\gamma_{0}$ also depends on the crack tip velocity (and temperature). We believe that the strong velocity dependence $v^{\alpha}(\alpha=0.6)$ they observe for $\gamma_{\text {eff }}$ results from a combination $\left(\alpha=\alpha_{1}+\alpha_{2}\right)$ of a velocity dependence of $[1+f(v)]$ (roughly $\alpha_{1} \approx 0.2$ ) and a velocity dependence of $\gamma_{0}(v)$ (roughly $\alpha_{2} \approx 0.4$ ). We note that thermal activation of the interfacial bond breaking is particular important for the weak bonds as involved in many adhesion studies, while for strong covalent bonds it is much less important. This explains why Gent in his study of the peeling of two SB rubber sheets, cross-linked by covalent (sulfur) bounds at the interface, found $\alpha=0.27$ in a large velocity range, in close agreement with the theory prediction $\alpha=\beta /(1+\beta)=0.26$.

\section{Appendix C. Radiative and damped detachment events}

We note that even for solids that are perfectly elastic, equation (10) is only approximately valid, because during pull-off some fraction of the stored elastic energy $U_{\mathrm{el}}$ will be lost as radiation of sound waves, rather than used to break interfacial bonds, see figure C.1. For a viscoelastic solid some fraction of $U_{\mathrm{el}}$ will also be lost during the viscoelastic deformations associated with the local detachment. Thus, of the order of $\alpha U_{\mathrm{el}}$ of the stored elastic energy will be lost where $\alpha \approx(\pi / 2) \tan \delta(\omega)$. In the present case $\tan \delta \approx 0.2$ (see figure 1), and we conclude that some fraction of the stored elastic energy will be lost due to the viscoelastic energy dissipation, which will contribute to the pull-off force. 
(a)

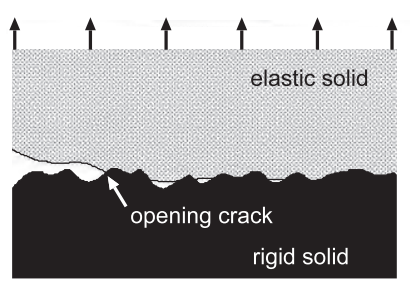

(b)

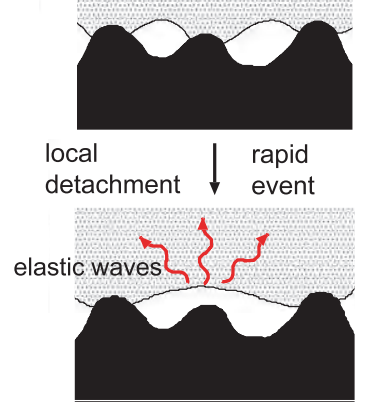

Figure C.1. (a) An opening crack during pull-off. (b) A local detachment event in front of the crack tip. An asperity contact region detaching rapidly with energy radiating inside the block rather than being used to break other asperity contact regions. For a viscoelastic solid, bulk energy dissipation may also occur during the detachment process, in particular if the frequency $\omega \approx(|E| / \rho)^{1 / 2} q$ (where $|E|=|E(\omega)|$ is the absolute value of the elastic modulus at a frequency $\omega, \rho$ is the rubber mass density and $q \approx 1 / D$, where $D$ is the diameter of the detached region) occurs in a region where $\tan \delta=\operatorname{Im} E(\omega) / \operatorname{Re} E(\omega)$ is large.

\section{References}

[1] Kendall K 2001 Molecular Adhesion and its Applications: The Sticky Universe (New York: Kluwer Academic)

[2] Chaudhury M K and Whitesides G M 1991 Langmuir 71013

[3] Mary P, Chateauminois A and Fretigny C 2006 J. Phys. D: Appl. Phys. 393665

[4] Persson B N J, Kovalev A, Wasem M, Gnecco E and Gorb S N 2010 Europhys. Lett. 9246001

[5] Guidoni G M, Schillo D, Hangen U, Castellanos G, Arzt E, McMeeking R M and Bennewitz R 2010 J. Mech. Phys. Solids $\mathbf{5 8} 1571$

[6] See, e.g. Persson B N J, Albohr O, Tartaglino U, Volokitin A I and Tosatti E 2005 J. Phys.: Condens. Matter 17 R1

[7] Fuller K N G and Tabor D 1975 Proc. R. Soc. A 345327

[8] Tobolsky A and Eyring H 1943 J. Chem. Phys. 11125

[9] Ghatak A, Vorvolakos K, She H, Malotky D L and Chaudhury M K 2000 J. Phys. Chem. B 1044018

[10] Brochard-Wyart F, de Gennes P G, Leger L, Marciano Y and Raphael E 1994 J. Chem. Phys. 989405

[11] Lake G J and Thomas A G 1967 Proc. R. Soc. A 300108

[12] Schallamach A 1963 Wear 6375

[13] Persson B N J and Volokitin A I 2006 Eur. J. Phys. E 2169

[14] Persson B N J 1995 Phys. Rev. B 5113568

[15] Persson B N J 1999 J. Chem. Phys. 1109713

[16] Peressadko A G, Hosoda N and Persson B N J 2005 Phys. Rev. Lett. 95124301

[17] Nguyen D T, Ramakrishna S, Fretigny C, Spenser N N, Le Chenadec Y and Chateauminois A 2013 Tribol. Lett. 49135

[18] Williams M L, Landel R F and Ferry J D 1955 J. Am. Chem. Soc. 773701

[19] Carbone G, Lorenz B, Persson B N J and Wohlers A 2009 Eur. Phys. J. E 29275

[20] See, e.g. Barabasi A L and Stanley H E 1995 Fractal Concepts in Surface Growth (Cambridge: Cambridge University Press)

[21] Lazic P and Persson B N J 2010 Europhys. Lett. 9146003

[22] Brörmann K, Burger K, Jagota A and Bennewitz R 2012 J. Adhes. 88589

[23] Krick B A, Vail J R, Persson B N J and Sawyer W G 2012 Tribol. Lett. 45185

[24] Persson B N J, Prodanov N, Krick B A, Rodriguez N, Mulakaluri N, Sawyer W G and Mangiagalli P 2012 Eur. Phys. J. E 351

[25] Heinrich G, Stuve J and Gerber G 2002 Polymer 43395

[26] Creton C and Lakrout H 2000 J. Polym. Sci. B 38965

[27] Carbone G and Mangialardi L 2004 J. Mech. Phys. Solids 521267

[28] Gent A N and Schultz J 1972 J. Adhes. 3281

[29] Maugis D and Barquins M 1978 J. Phys. D: Appl. Phys. 111989

[30] Gent A N 1996 Langmuir 124492

[31] Persson B N J and Brener E A 2005 Phys. Rev. E 71036123

[32] Persson B N J, Albohr O, Heinrich G and Ueba H 2005 J. Phys.: Condens. Matter 17 R1071

[33] Lorenz B, Persson B N J, Dieluweit S and Tada T 2011 Eur. Phys. J. E 34129

[34] Johnson K L, Kendall K and Roberts A D 1971 Proc. R. Soc. A 324301

[35] Johnson K L 1966 Contact Mechanics (Cambridge: Cambridge University Press)

[36] Greenwood J A and Johnson K L 1981 Phil. Mag. A 4369 Greenwood J A 2004 J. Phys. D: Appl. Phys. 372557

[37] Thanawala S K and Chaudhury M K 2000 Langmuir 161256

[38] Persson B N J 2007 J. Adhes. Sci. Technol. 211145

[39] Persson B N J 2002 Eur. Phys. J. E 8385

[40] Persson B N J, Sivebaek I M, Samoilov V N, Zhao K, Volokitin A I and Zhang Z 2008 J. Phys.: Condens. Matter 20395006

[41] Persson B N J, Albohr O, Creton C and Peveri V 2004 J. Chem. Phys. 1208779

[42] Kroner E, Maboudian R and Arzt E 2010 Adv. Eng. Mater. 12398

[43] Silberzan P, Perutz S, Kramer E J and Chaudhury M K 1994 Langmuir 102455

[44] She H, Malotky D and Chaudhury M K 1998 Langmuir 143090

[45] Kröger M, Popp K and Kendziorra N 2004 Mach. Dyn. Probl. 2879

[46] Moldenhauer P, Nepp R and Kröger M 2011 Plast. Rubber Compos. $\mathbf{4 0} 169$

[47] Kröger M 2011 Adhäsioneffekte an Normalkontakten mit Elastomeren (Tagungsband) vol 52 (Göttingen: Tribologie-Fachtagung GfT Band I) pp S.2/1-S.2/15

[48] Baytekin B, Baytekin H T and Grzybowski B A 2012 J. Am. Chem. Soc. 1347223

[49] Mott P H, Roland C M and Corsaro R D 2002 J. Acoust. Soc. Am. 1111782

[50] Shull K 2002 Mater. Sci. Eng. R 361 